\title{
Identification and determination of aroma components of some juice and its blends
}

\author{
Mohamed K. Abd el-Aziz ${ }^{1,2}$, Ashraf M. Sharoba ${ }^{1}$, Ahmed E. El-Desouky ${ }^{1}$, Hassan H. Khalaf ${ }^{1}$ and Osama M. Morsy ${ }^{3}$ \\ ${ }^{1}$ Department of Food Technology, Faculty of Agriculture, Benha University, Qaluobia, Egypt. \\ ${ }^{2}$ Kaha Company for preserved foods, Holding Company for food industries, Ministry of supplyand internal trade, Egypt. \\ ${ }^{3}$ Arab Academy for Science, Technology \& Maritime Transport, Arab League.
}

Corresponding author: ashraf_sharoba@yahoo.com

\begin{abstract}
In this study, the chromatographic analysis method was used to determine the components responsible for the flavor in some types of juices and beverages in Egypt. The components of the flavor were also estimated in mango juice, orange juice, apricot juice, carrot juice and kaki juice after the addition of pectinase and cellulose enzymes with a concentration of $0.1 \%$ to determine the effect of these enzymes on the flavor components of this juice. And also prepared 4 mixtures including mango juice with orange juice, mango juice with carrot juice, mango juice with apricot juice and mango juice with kaki juice mixing rate 80:20\% respectively. Flavor components were estimated in 11 samples. The results showed that the product of mango juice contained propane, 2-methoxycontent of 21.86 and apricot juice on 9-Octadecenoic acid (Z)with 14.59 and orange juice on compound ethane, 1,1'-oxybis-with 79.36 and kaki juice on ethane, 1,1'-oxybis compound with 84.39 and carrot juice on 9octadecenoic acid (Z)-compound by 40.31. The addition of pectinase and celluloseal enzymes to mango juice increased 9-Octadecenoic Acid (Z)- by 86.88 and 96.29 respectively. And contain mango juice mixed with orange juice on 9-Octadecenoic acid (Z) compound with 54.91 and mango juice mixture juice carrots on the ethane, 1,1'oxybis compound 70.35 and mango juice mixed with kaki juice ethane, 1,1'-oxybis on the compound 89.07 and mango juice mixed with apricot juice ethane, 1,1'-oxybis- on the compound 89.07.
\end{abstract}

Key words: Aroma chemical composition; aroma components, Mango juice, Orange juice, Apricot juice, Carrots juice, Juice blends.

\section{Introduction}

Fruits produce a wide range of volatile organic compounds that impart their characteristically distinct aromas and contribute to unique flavor characteristics. Fruit aroma and flavor characteristics are of key importance in determining consumer acceptance in commercial fruit markets based on individual preference. Fruit producers, suppliers and retailers traditionally utilize and rely on human testers or panels to evaluate fruit quality and aroma characters for assessing fruit salability in fresh markets, Baietto and Wilson (2015).

Flavor study in different foods has been of increasing interest due to its relationship with the quality of product. In the case of fruits, aroma is one of the most appreciated characteristics. Fruit flavor is particularly sensitive to compositional alteration. The volatile compounds that form the fruit flavor are produced through metabolic pathways during ripening, harvest, post - harvest and storage and depend on many factors related to the species, varity and type of technological treatment (Rizzolo et al., 2002)

Aroma profiles of fruit juices usually comprise a mixture of a large number of volatile organic compounds. The individual aroma components differ according to their molecular structure, which in turn defines the solubility, boiling point, and the volatility of each type of compound, Ramteke et al., (1990) .

Fruit aromas consist of a complex mixture of VOCs whose composition is specific to plant species and fruit variety. Although different fruits often share many aromatic characteristics, each fruit has a distinctive aroma that depends upon the combination of volatiles, the concentration and the perception threshold of individual volatile compounds. The most important aroma compounds include amino acidderived compounds, lipid-derived compounds, phenolic derivatives, and mono- and sesquiterpenes. Although fruit aromas are generally complex mixtures of a wide range of compounds, Tucker (1993).

Fruits produce and release a wide variety of Volatile Organic Compounds (VOCs) that make up their characteristic aromas with esters, terpenoids, lactones and derivatives of amino acids, fatty-acids and phenolic compounds being the dominant classes of organic volatiles represented in fruit aromas, Schwab et al., (2008).

Mango (Mangifera indica L.) popularly known as 'The King of Fruits' and currently ranks fifth in total production among major fruit crops worldwide and is grown commercially in more than 87 countries, Tharanathan, (2006).

Mango (Mangifera Indica Cv. Chokanan) is one of the most important and popular commercial tropical fruits in the World. In the processing of mango products like mango pulp and amchur (dried, powdered unripe mango), mango peel is a major byproduct ( Ajila et al., 2007 ) of mango juice industry.

Orange juice is one of themajor traded commodities throughout the world, and themostwidely consumed fruit juice. It comprises $46 \%$ of the worldwide fruit juice consumption, amounting to 18,449 million L in 2013 (Markstrat, 2013). 
Carrots (Daucus carota L.) are one of the most widely consumed vegetables because of their characteristic flavor and health benefits. Carrots are the major vegetable source of provitamin A, and they play a central role in the increased interest in healthy food as well as the demand for more vegetables containing various health-related compounds (Theodosiou and others 2010).

Apricot fruit (Prunus armeniaca) is native from China and is widely adapted to Mediterranean conditions. It is consumed around the world due to its pleasant and delightful aroma, which has been previously studied, Azodanlou et al., (2003).

Persimmon (Diospyros kaki L.) is an important fruit crop and approximately 4.6 million tons of persimmon fruit were produced globally in 2013 (FAO., 2013).

The ability of acidic (AcW) and alkaline electrolyzed waters (AlW) to improve the flavour of persimmon (Diospyros kaki L.) wine was evaluated. Wines made with AcW (WAcW) were significantly better than wines made with AlW or pure water (PW) in aroma, taste, and colour.( Zhu., 2016)

Khakimov et al., (2016) studies that, tropical fruits contribute significantly to the total fruit intake worldwide. However, their metabolomes have not yet been investigated comprehensively, as most previous studies revealed only volatile and bulk compositions.

Qiao et al., (2008) reported that, gas chromatography-mass spectrometry (GC-MS) and gas chromatographyolfactometry (GC-O) were used to determine the aromatic composition and aroma active compounds of fruit juice and peel oil of Jinchen sweet orange fruit. The aromatic compositions of fruit juice were more complex than that of peel oil. Ethyl butanoate, $\beta$-myrcene, octanal, linalool, $\alpha$-pinene, and decanal were found to be responsible for the aromatic notes in fruit juice and peel oil. Nineteen components have been perceived only in the juice and ten compounds were described as aromatic components of only the peel oil by the panelists. These differences lead to the different overall aroma between fruit juice and peel oil.

Musharraf et al., (2016) studies that, a quantitative method was developed based on gas chromatography triple quadrupole mass spectrometry (GC-QQQ-MS) for the analysis of aroma component of mango sap (latex) in nine Pakistani varieties that are Anmol, Began pali, Badami, Caroba, Chaunsa, Lal patra, Neelum, Sohnara and Tota pari.

Singh et al.,(2014) determine the influence of postharvest vapour heat treatment (VHT) on qualitative and quantitative measurement of aroma volatiles during fruit ripening in mango (cv. Chausa) using gas chromatography-mass spectrometry (GCMS) .

Mastello et al ., (2015) Odour-active compounds present in pasteurised orange juice were identified by gas chromatography- olfactometry (GC-O) employing heart-cut multidimensional GC techniques with olfactometry $(\mathrm{O})$ and mass spectrometry $(\mathrm{H} / \mathrm{C}$ MDGC-O/MS) and comprehensive two-dimensional gas chromatography-accurate mass time-offlight MS (GC $\times$ GC-accTOFMS). Headspace solid phase microextraction sampling proved to be qualitatively adequate for the analysis of pasteurised orange juice .

Aroujalian et al., (2007) showed that pervaporative recovery of volatile aroma compounds from orange juice was studied to determine the influence of various operating parameters such as feed flow rate which is correspondent with Reynolds numbers of 500, 1000, 1500, 2000, and 2500, feed temperature $(25,40$, and $50 \circ \mathrm{C})$ and permeate pressure $(1,10,20,30$, and $40 \mathrm{mmHg})$ on flux and selectivity.

Selli et al., (2004) studies that, the volatile flavour components of orange juice obtained from the $\mathrm{cv}$. Kozan oranges were investigated. Flavour components were extracted by using Amberlite XAD2 polymeric resin and eluted by pentane/ dichloromethane solvent and then analysed by gas chromatography-flame ionization detection (GCFID) and gas chromatography-mass spectrometry (GCMS). Thirty-four components, including seven esters, two aldehydes, five alcohols, five terpenes, twelve terpenols, and three ketones were identified and quantified. The major flavour components were linalool, limonene, $\beta$-phellandrene, terpinene-4-ol and ethyl 3-hydroxy hexanoate.

Solı's et al., (2007) studies tha, the volatile fraction of eight varieties of apricot were analyzed using simultaneous distillation extraction (SDE), solid phase extraction (SPE) with reverse phase (C18), liquid-liquid extraction (LLE) and headspace-solid phase microextraction (HS-SPME). The free aroma compounds were identified by GC-MS, finding common compounds such as linalool, $\alpha$-terpineol, $\beta$ ionone and $\Upsilon$-decalactone and specific compounds due to the extraction method used. On the other hand, Solis et al., (2007) obtained from eight varieties of apricots (Prunus armeniaca) were compounds in SPME (hexanol, limonene, 2-hexenal, 6- metil-5hepten-2-one, linalool, 3,7-dimetil-1,6-octadiene, $\beta$ ionone, $\Upsilon$-decalactone), and with five aroma compounds in LLE (limonene, linalool, 1,3dimethylcyclohexanol, cyclohexylisotiocianate, $\beta$ ionone). These aroma compounds served to recognize and classify all the analyzed varieties.

Riu-Aumatell et al., (2004) described that, a rapid evaluation of volatile profiles of several commercial fruit juices (pear, apricot and peach) by headspace-solid phase microextraction and gas chromatography/mass spectrometry (HS-SPME and GC/MS). In addition, this is the first study to report the detection of several norisoprenoids (mainly naphthalenes) that characterised apricot and peach juices. Moreover, by means of volatile compounds it could be possible to distinguish between juices of organic and conventional production and juices with flavourings additioned. 
Kjeldsen et al. , (2001) showed that, dynamic headspace sampling was used to collect aroma compounds from raw samples of four carrot (Daucus carota L.) cultivars (Brasilia, Duke, Fancy, and Cortez). The collected volatiles were analyzed by capillary GC-FID and GC-MS using large-volume cool on-column injection (LVI-COC). Of the 36 compounds identified, 6 had not been previously detected in carrots. Significant differences between the carrot cultivars were found for 31 of the identified volatiles as well as for total monoterpenes, sesquiterpenes, on the other hand, the loss among major monoterpenes in the concentrated samples varied from $16 \%$ for p-cymene to $>40 \%$ for R-pinene as compared to nonconcentrated samples. The loss among high-boiling sesquiterpenes varied from not detectable ( $\beta$-caryophyllene, $\alpha$-humulene, and caryophyllene oxide) to approximately $7 \%$ for (E)and $(\mathrm{Z})$ - $\gamma$-bisabolene.

Wang et al. , (2011) showed that, An aroma extract of persimmon fruit (Diospyros kaki L., var. Triumph) was obtained by hydrodistillation under vacuum followed by solid phase extraction. Gas chromatography-mass spectometry (GC-MS) analysis of the extract led to the positive identification of 50 compounds, among which aldehydes emerged as the most important class of volatile compounds. Thirty-two compounds were determined to have aroma-impact, by gas chromatography-olfactometry analysis of the aroma extract. The six most intense aroma-impact compounds were methional, (E)-2hexenal, phenylacetaldehyde, (E,Z)-2,6-nonadienal, hexanal and Furaneol.

Wang et al. , (2011) investigation that, persimmon flavour, we applied recent improvements in volatiles isolation, gas chromatography-mass spectrometry (GC-MS) and gas chromatographyolfactometry (GC-O) analyses to characterize the volatiles and aroma-impact compounds of persimmon fruit available in a US local food market.

Kjeldsen et al. , (2001) reported that, gas chromatogram of a carrot headspace sample is shown a total of 44 volatile compounds were repeatedly detected and quantified in the different carrot cultivars. Thirty-six of these were identified by comparison of their mass spectral data with those from authentic compounds and/or mass spectra suggested by the NIST database (33) and GC retention indices.

Aroma of apricots (Prunus armeniaca) were compounds commonly found in all the analyzed varieties was established: hexilacetate, limonene, 6methyl-5-hepten-2-one, mentone and trans-2-hexenal as the most common and furthermore, linalool, -ionone and 3,7-dimethyl-1,6-octadiene Guillot $\boldsymbol{e t}$ al., (2006).

Pectins are complex high-molecular-mass glycosidic macromolecules that contribute to complex physiological processes such as cell differentiation or cell growth, being largely responsible for the structural integrity and cohesion of plant tissues (Sharma et al., 2006; Nigam and Pandey, 2009).

Cellulose is a structural carbohydrate and one of the cell-wall constituents of plants. It is considered a complex sugar, used for protection and structure of the plant, where the cellulose fibers are embedded in a matrix of other structural biopolymers, primarily hemicellulose and lignin (Sharma et al., 2014).

\section{Materials And Methods Materials:}

The raw materials used throughout this study for production juice nectar and nectar blends were:

1. Mango and apricot pulp were obtained from Kaha Company, Kaha, Qalyabia governorate, Egypt.

2. Carrot, kaki and orange were obtained from local market, Benha, Qalyabia governorate, Egypt.

3. Cellulase and bectinase enzymes were obtained from Novo Nordisk, Switzerland.

Technological treatments:

\section{Preparation of nectar blends:}

The mango juice and fresh juices were blended together as follows:

Blend (1):80 gm mango juice and/or (20 gm orange, 20 gm apricot, 20 gm kaky, and 20 gm carrot juices). Nectar was prepared as recommended methods described by Sharoba (2007) from 25\% (mango, orange, apricot, kaky and carrot) juices $+75 \%$ (Sugar Solution) to get total soluble solids (16-18\%), pH 3.5. The $\mathrm{pH}$ was adjusted to 3.5 by adding citric acid as $50 \%$ (W/V) solutions according to AOAC (2016).

\section{Methods:}

\section{Determination of volatile components: \\ Extraction of volatile compounds:}

Three hundred grams of pulp were homogenized and mixture with (1 L of water) then distilled by a simultaneous steam distillation. The volatile aroma compounds were extracted using (dichloromethane solvent, $200 \mathrm{ml}$.). The aroma extract was dried over anhydrous sodium sulfate and concentrated using by rotary evaporator, the obtained concentrates were analyzed using gas chromatography-mass spectrometry, Abd el- hafez(2009).

\section{Combined gas chromatography-mass spectrometry:}

GC-MS analysis was performed with an Aglient 6890 gas chromatograph (Made in England) equipped with an Aglient mass spectrometric detector, with a direct capillary interface and fused silica capillary column HP-5MS (30 m X $320 \mu \mathrm{m}$ X $0.25 \mu \mathrm{m}$ film thickness). Samples were injected under the following conditions:

Helium was used as carrier gas at approximately $1.0 \mathrm{ml} / \mathrm{min}$., pulsed splitless mode. The solvent delay was $3 \mathrm{~min}$. and the injection size was $1.0 \mu \mathrm{l}$. The GC temperature program was started at $40^{\circ} \mathrm{C}(3 \mathrm{~min})$ then 
elevated to $260^{\circ} \mathrm{C}$ at rate of $8^{\circ} \mathrm{C} / \mathrm{min}$. the detector and injector temperature were set at 280 and $250^{\circ} \mathrm{C}$, respectively. Wiley mass spectral data base was used in the identification of the separated peaks.

\section{Results And Discussion}

Data presented in Table (1) showed that determination of mango juice by $\mathrm{GC} / \mathrm{MS}$ chromatography analysis compounds, which could be classified mainly as aldehyde ketones, alcohols, fatty acids and hydrocarbons. Aldehydes and alcohols were the most representative chemical fractions and contents were: the highest propane, 2-methoxy(21.86), 1,2-ethanediol,monoacetate(10.86), ethane, 1,1'-oxybis-(9.82), docosane,(5.33),2,2-dimethoxy5,5-dimethyl-1,3,4-.deta.(3) oxadiazoline (3.80), ethyl2-(1'-hydroxy-1'-methylethyl)-5,6, trimethyl-3,4-hep tadienoate, (3.75), acetic acid, methoxy-, methyl ester,(3.74), ethane, 1,2-diethoxy(3.57) and 2-butanol (3.30). on the other hand, propane, 2-methoxy-(0.01), 2-butanol (0.22), acetic acid, methyl ester (0.28), formic acid, ethyl ester, ( 0.33), propanoic acid,2-hydroxy-2-methyl-,methyl ester, ( 0.35$)$, ethane, 1,1'-oxybis- ( 0.43$)$ and heptadecane, 9-hexyl- ( 0.65$)$ were the lowest contents compounds.

Results on (Table 1) were in agreement with those reported by Zhu, et al (2014) the volatile profile of the persimmon wines and juices was determined by GCMS with the aid of SBSE, leading to the identification of 50 and 60 compounds, respectively. Among these compounds, only 26 detected compounds showed the OAV above 1 in all persimmon wines. Abu el- maaty (2012) found that, mango juice found high concentration Myrcene $38.58 \%$ - limonene $11.68 \%$ Ocimene $10.56 \%$ and less concentration1-Butanol $0.01 \%$ and Ethyl isobutyrate $02 \%$.

The main pattern of aroma released and formed from the yeast employed in the vinification of persimmon must was clear: the strain was able to produce fermentative ethyl esters which were responsible for fresh fruit attributes. In our study, persimmon wines produced by strains IFFI 1363 and D254 were characterised by persimmon, aroma harmony, fruity, fusel and taste balanced, fullness and hedonic scale. Therefore, the two yeast strains could be used as starter culture for persimmon wine production. In addition, based on the characteristics of the produced persimmon wine, persimmon fruits showed great application potential in the production of fermented beverages.

Data presented in Table (2) showed that determination of apricot juice by $\mathrm{GC} / \mathrm{MS}$ chromatography analysis compounds, which could be classified mainly as aldehyde ketones, alcohols, fatty acids and hydrocarbons. Aldehydes and alcohols were the most representative chemical fractions and contents were the highest 9-octadecenoic acid (z)( 14.59), ethane, 1,1'-oxybis-(13.16), 9- octadecenoic acid (z)-, methyl ester (11.31), 10undecenoic acid,methyl ester (10.56), [1,1'bicyclopropyl]-2-octanoic acid, 2'-hexyl-, methyl ester ( 10.15), 1-hexadecanol, 2-methyl- (9.75), 9Hexadecenoic acid (6.25), d-glycero-d-galactoheptose (6.12) and 6-nonenoic acid, methyl ester ( 5.28) . on the other hand, 1-deoxy-d-mannitol (2.24), 2-ethoxyethanol (2.60), 6-nonenoic acid, methyl ester ( 3.09) and z-(13,14-epoxy)tetradec-11en-1-ol acetate (3.28). were the lowest contents compounds.

Results on (Table 2) were in agreement with those reported by Solís-Solís,et al (2007) The aroma of a food is not related to the total concentration of volatile compounds, but to the aromatic compounds characteristics of the fruit impact compound that are in that volatile fraction. Results on (Table 2) were in agreement with those reported by Evrendilek,(2016) given the mean values of the 73 aroma active compounds across the treatment times, the initial mean values of the 10 compounds on average increased significantly for apricot (six) and peach (four) nectars, while the 33 compounds decreased significantly for peach (24) and apricot (eight) nectars, and sour cherry juice (one). Abu el- maaty (2012) found that, apricot juice observed the highest concentration 1- Butanol 13.28\% ester compound was less concentration Ethyl dodecanoate $0.07 \%$.

Data presented in Table (3) showed that determination of orange juice by $\mathrm{GC} / \mathrm{MS}$ chromatography analysis compounds, which could be classified mainly as aldehyde ketones, alcohols, fatty acids and hydrocarbons. Aldehydes and alcohols were the most representative chemical fractions and contents were the highest ethane, 1,1'-oxybis-(79.36), 9-octadecenoic acid (z)( 16.94), oleic acid(12.01), [1,1'-bicyclopropyl]-2-octanoic acid, 2'-hexyl-, methyl ester( 7.31) and 9,10,11-octadecenoic acid (z)-, methyl ester (5.63). on the other hand, ethane, 1,1,2-trimethoxy-(0.01), 13-tetradecynoic acid, methyl ester(0.19), 9-octadecenoic acid, (2-phenyl1,3-dioxolan-4-yl)methyl ester, cis( 0.22), 7nonenoic-7,8-d2 acid, methyl ester( 0.42) and 10undecenoic acid, methyl ester( 0.50) were the lowest contents compounds. .

Results on (Table 3) were in agreement with those reported by Mastello,et al (2015) demonstrated an efficient analytical approach using multidimensional techniques of MDGC and GC $\times$ GC coupled with olfactometry and MS to assess pasteurised orange juice aroma. The $\mathrm{GC}-\mathrm{O}$ DF technique allowed successful screening of the most significant odour impact zones (according to $\mathrm{DF} \geq 3$ ) for volatile composition in pasteurised orange juice. Four aldehydes (hexanal, heptanal, octanal, citral), 2 esters (ethyl butanoate, methyl hexanoate), and 4 monoterpenes ( $\alpha$ pinene, D-limonene, linalool, $\alpha$-terpineol) were confirmed in accordance with olfactometry assessment in the processed juice. Abu el- maaty (2012) found that, orange juice found compound D-limonene $45.57 \%$, ethanol $12.67 \%$. The composite Alaladheady found Neral $12 \%$ the compound Alkithoni Carvone $0.35 \%$. 
Table 1. Identification and determination of mango juice by GC/ MS chromatography analysis:

\begin{tabular}{|c|c|c|c|c|c|}
\hline $\begin{array}{l}\text { Peak } \\
\text { No. }\end{array}$ & R.t* & Compound name & $\begin{array}{c}\text { Area } \\
\%\end{array}$ & $\begin{array}{c}\text { Molecular } \\
\text { Weight }\end{array}$ & $\begin{array}{c}\text { Molecular } \\
\text { formula }\end{array}$ \\
\hline 1 & 2.03 & DIMETHOXYPROPANE & 1.40 & 104 & $\mathrm{C}_{5} \mathrm{H}_{12} \mathrm{O}_{2}$ \\
\hline 2 & 2.30 & ETHANE, 1,1'-OXYBIS- & 9.82 & 74 & $\mathrm{C}^{4} \mathrm{H}_{10} \mathrm{O}$ \\
\hline 3 & 2.54 & 1,2-ETHANEDIOL,MONOACETATE & 10.86 & 104 & $\mathrm{C}_{4} \mathrm{H}_{8} \mathrm{O}_{3}$ \\
\hline 4 & 2.68 & PROPANE, 2-METHOXY- & 21.86 & 74 & $\mathrm{C}_{4} \mathrm{H}_{10} \mathrm{O}$ \\
\hline 5 & 2.96 & 2-OXIRANYLMETHYL 2 METHYLACRYLATE \# & 1.44 & 142 & $\mathrm{C}_{7} \mathrm{H}_{10} \mathrm{O}_{3}$ \\
\hline 6 & 3.21 & Ethane, 1,2-diethoxy- & 3.57 & 118 & $\mathrm{C}_{6} \mathrm{H}_{14} \mathrm{O}_{2}$ \\
\hline 7 & 3.54 & Hexadecanoic acid,1,5-pentanediyl ester & 1.27 & 580 & $\mathrm{C}_{37} \mathrm{H}_{72} \mathrm{O}_{4}$ \\
\hline 8 & 3.65 & ACETAMIDE & 1.13 & 59 & $\mathrm{C}_{2} \mathrm{H}_{5} \mathrm{NO}$ \\
\hline 9 & 3.74 & DOCOSANE & 5.33 & 310 & $\mathrm{C}_{22} \mathrm{H}_{46}$ \\
\hline 10 & 3.81 & Acetic acid, methoxy-, methyl ester & 3.74 & 104 & $\mathrm{C}_{4} \mathrm{H}_{8} \mathrm{O}_{3}$ \\
\hline 11 & 3.97 & BUTANOIC ACID,2-HYDROXY-, METHYL ESTER & 0.38 & 118 & $\mathrm{C}_{5} \mathrm{H}_{10} \mathrm{O}_{3}$ \\
\hline 12 & 4.09 & $\begin{array}{l}\text { ETHYL2-(1'-HYDROXY-1'-METHYLET HYL)- } \\
\text { 5,6,6-TRIMETHYL-3,4-HEP TADIENOATE }\end{array}$ & 3.75 & 254 & $\mathrm{C}_{15} \mathrm{H}_{26} \mathrm{O}_{3}$ \\
\hline 13 & 4.14 & $\begin{array}{l}\text { 2,2-DIMETHOXY-5,5-DIMETHY L-1,3,4- } \\
\text {.DETA.(3)-OXADIAZOLINE }\end{array}$ & 3.80 & 160 & $\mathrm{C}_{6} \mathrm{H}_{12} \mathrm{~N}_{2} \mathrm{O}_{3}$ \\
\hline 14 & 4.36 & BUTANOIC ACID,2,3-DIMETHYL- & 2.28 & 116 & $\mathrm{C}_{6} \mathrm{H}_{12} \mathrm{O}_{2}$ \\
\hline 15 & 4.45 & $\begin{array}{l}\text { METHYL-(3R)-(-)-3-ETHYL-5-O } \\
\text { XOPENTANOATE }\end{array}$ & 2.23 & 158 & $\mathrm{C}_{8} \mathrm{H}_{14} \mathrm{O}_{3}$ \\
\hline 16 & 4.52 & FORMIC ACID, ETHYL ESTER & 0.33 & 74 & $\mathrm{C}_{3} \mathrm{H}_{6} \mathrm{O}_{2}$ \\
\hline 17 & 4.62 & PROPANE, 2-METHOXY- & 0.01 & 74 & $\mathrm{C}_{4} \mathrm{H}_{10} \mathrm{O}$ \\
\hline 18 & 5.46 & Heptadecane, 9-hexyl- & 0.65 & 324 & $\mathrm{C}_{23} \mathrm{H}_{48}$ \\
\hline 19 & 5.60 & Acetic acid, methyl ester & 0.28 & 74 & $\mathrm{C}_{3} \mathrm{H}_{6} \mathrm{O}_{2}$ \\
\hline 20 & 5.80 & 2-Butanol & 0.22 & 74 & $\mathrm{C}_{4} \mathrm{H}_{10} \mathrm{O}$ \\
\hline 21 & 6.58 & $\begin{array}{l}\text { PROPANOIC ACID,2-HYDROXY-2-METHYL- } \\
\text {,METHYL ESTER }\end{array}$ & 0.35 & 118 & $\mathrm{C}_{5} \mathrm{H}_{10} \mathrm{O}_{3}$ \\
\hline 22 & 7.43 & ETHANE, 1,1'-OXYBIS- & 0.43 & 74 & $\mathrm{C}_{4} \mathrm{H}_{10} \mathrm{O}$ \\
\hline 23 & 7.76 & BUTYL ESTER OFHYDROXYACETIC ACID & 2.12 & 132 & $\mathrm{C}_{6} \mathrm{H}_{12} \mathrm{O}_{3}$ \\
\hline 24 & 7.85 & PENTOSE & 1.26 & 150 & $\mathrm{C}_{5} \mathrm{H}_{10} \mathrm{O}_{5}$ \\
\hline 25 & 9.49 & CIS-INOSITOL & 2.50 & 180 & $\mathrm{C}_{6} \mathrm{H}_{12} \mathrm{O}_{6}$ \\
\hline 26 & 10.71 & BUTANE, 2-METHOXY- & 1.20 & 88 & $\mathrm{C}_{5} \mathrm{H}_{12} \mathrm{O}$ \\
\hline 27 & 13.43 & NONANAL & 2.15 & 142 & $\mathrm{C}_{9} \mathrm{H}_{18} \mathrm{O}$ \\
\hline 28 & 13.90 & Gibberellic acid & 1.15 & 346 & $\mathrm{C}_{19} \mathrm{H}_{22} \mathrm{O}_{6}$ \\
\hline 29 & 16.41 & 2-BUTANOL & 3.30 & 74 & $\mathrm{C}_{4} \mathrm{H}_{10} \mathrm{O}$ \\
\hline 30 & 19.49 & 2-DECENAL, (E)- & 2.46 & 154 & $\mathrm{C}_{10} \mathrm{H}_{18} \mathrm{O}$ \\
\hline 31 & 21.76 & 2,4-DODECADIENAL, (E,E)- & 2.85 & 180 & $\mathrm{C}_{12} \mathrm{H}_{20} \mathrm{O}$ \\
\hline 32 & 26.89 & 11,14-Octadecadiynoic acid,methyl ester & 1.50 & 290 & $\mathrm{C}_{19} \mathrm{H}_{30} \mathrm{O}_{2}$ \\
\hline 33 & 30.39 & 1-TRIDECANOL & 1.18 & 200 & $\mathrm{C}_{13} \mathrm{H}_{28} \mathrm{O}$ \\
\hline 34 & 31.81 & 9-OCTADECENOIC ACID (Z)- & 1.50 & 282 & $\mathrm{C}_{18} \mathrm{H}_{34} \mathrm{O}_{2}$ \\
\hline 35 & 33.39 & Oleic Acid & 1.12 & 282 & $\mathrm{C}_{18} \mathrm{H}_{34} \mathrm{O}_{2}$ \\
\hline
\end{tabular}

*R.t, retention time (min).

(values expressed as relative area percentage to total identified compounds). 


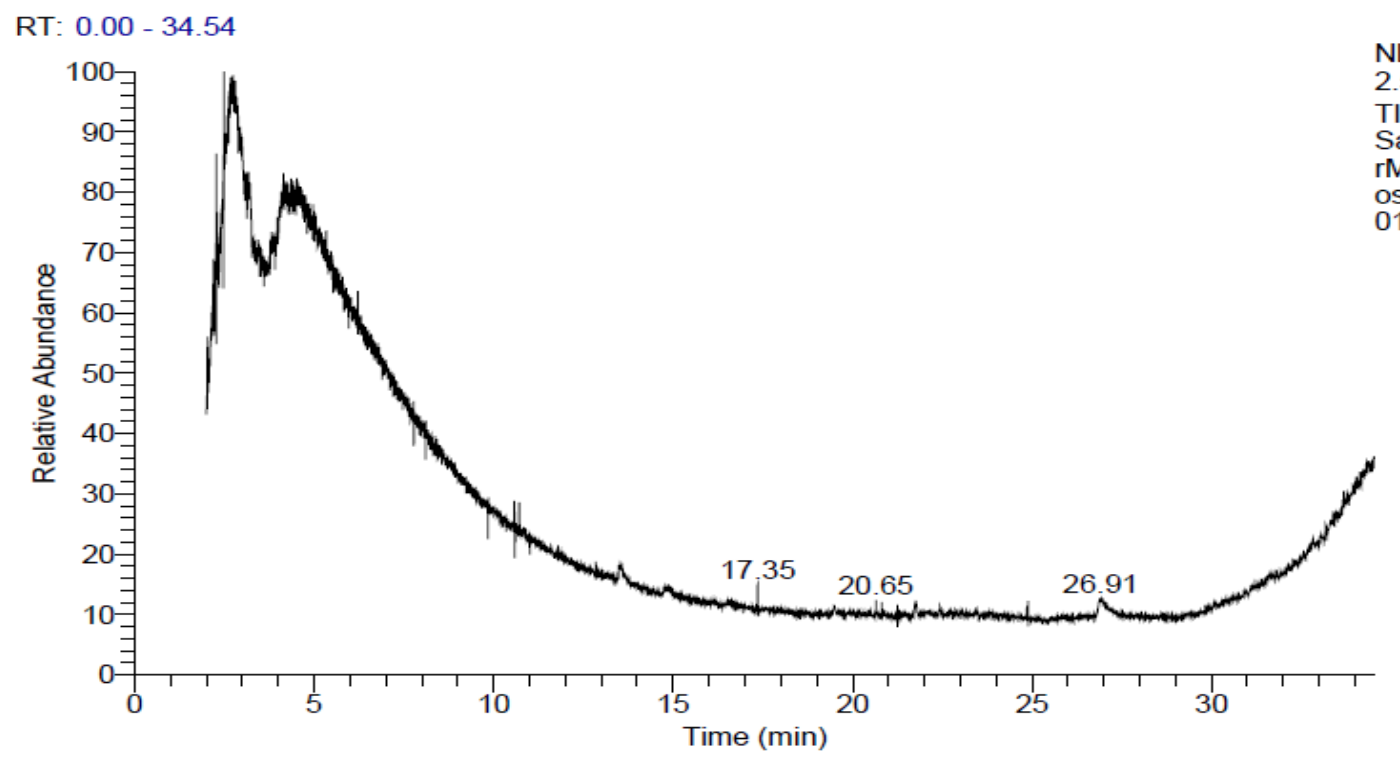

Fig (1) Identification and determination of mango juice by GC/ MS chromatography analysis

Table 2. Identification and determination of apricot juice by GC/ MS chromatography analysis:

\begin{tabular}{|c|c|c|c|c|c|}
\hline $\begin{array}{l}\text { Peak } \\
\text { No. }\end{array}$ & R.t* & Compound name & Area \% & $\begin{array}{l}\text { Molecular } \\
\text { Weight }\end{array}$ & $\begin{array}{l}\text { Molecular } \\
\text { formula }\end{array}$ \\
\hline 1 & 2.35 & ETHANE, 1,1'-OXYBIS- & 13.16 & 74 & $\mathrm{C}_{4} \mathrm{H}_{10} \mathrm{O}$ \\
\hline 2 & 7.12 & 6-Nonenoic acid, methyl ester & 5.28 & 170 & $\mathrm{C}_{10 \mathrm{H}_{18} \mathrm{O}_{2}}$ \\
\hline 3 & 8.01 & $\begin{array}{l}\text { 10-UNDECENOIC ACID,METHYL } \\
\text { ESTER }\end{array}$ & 10.56 & 198 & $\mathrm{C} 12 \mathrm{H} 22 \mathrm{O} 2$ \\
\hline 4 & 10.07 & 6-NONENOIC ACID, METHYL ESTER & 3.09 & 170 & $\mathrm{C} 10 \mathrm{H} 18 \mathrm{O} 2$ \\
\hline 5 & 1.83 & 2-ETHOXYETHANOL & 2.60 & 90 & $\mathrm{C} 4 \mathrm{H} 10 \mathrm{O} 2$ \\
\hline 6 & 15.28 & d-Glycero-d-galacto-heptose & 6.12 & 210 & C7H14O7 \\
\hline 7 & 19.32 & 1-Deoxy-d-mannitol & 2.24 & 166 & C6H14O5 \\
\hline 8 & 23.70 & $\begin{array}{l}{[1,1 \text { '-Bicyclopropyl]-2-octanoic acid, 2'- }} \\
\text { hexyl-, methyl ester }\end{array}$ & 10.15 & 322 & C21H38O2 \\
\hline 9 & 29.06 & 1-HEXADECANOL, 2-METHYL- & 9.75 & 256 & $\mathrm{C} 17 \mathrm{H} 36 \mathrm{O}$ \\
\hline 10 & 29.17 & $\begin{array}{l}\text { 9-OCTADECENOIC ACID (Z)-,METHYL } \\
\text { ESTER }\end{array}$ & 11.31 & 296 & С19H36O2 \\
\hline 11 & 30.68 & Z-(13,14-Epoxy)tetradec-11-en-1-ol acetate & 3.28 & 268 & $\mathrm{C} 16 \mathrm{H} 28 \mathrm{O} 3$ \\
\hline 12 & 31.95 & 9-Hexadecenoic acid & 6.25 & 254 & $\mathrm{C} 16 \mathrm{H} 30 \mathrm{O} 2$ \\
\hline 13 & 34.40 & 9-OCTADECENOIC ACID (Z) & 14.59 & 282 & $\mathrm{C} 18 \mathrm{H} 34 \mathrm{O} 2$ \\
\hline
\end{tabular}

*R.t, retention time (min).

(values expressed as relative area percentage to total identified compounds). 


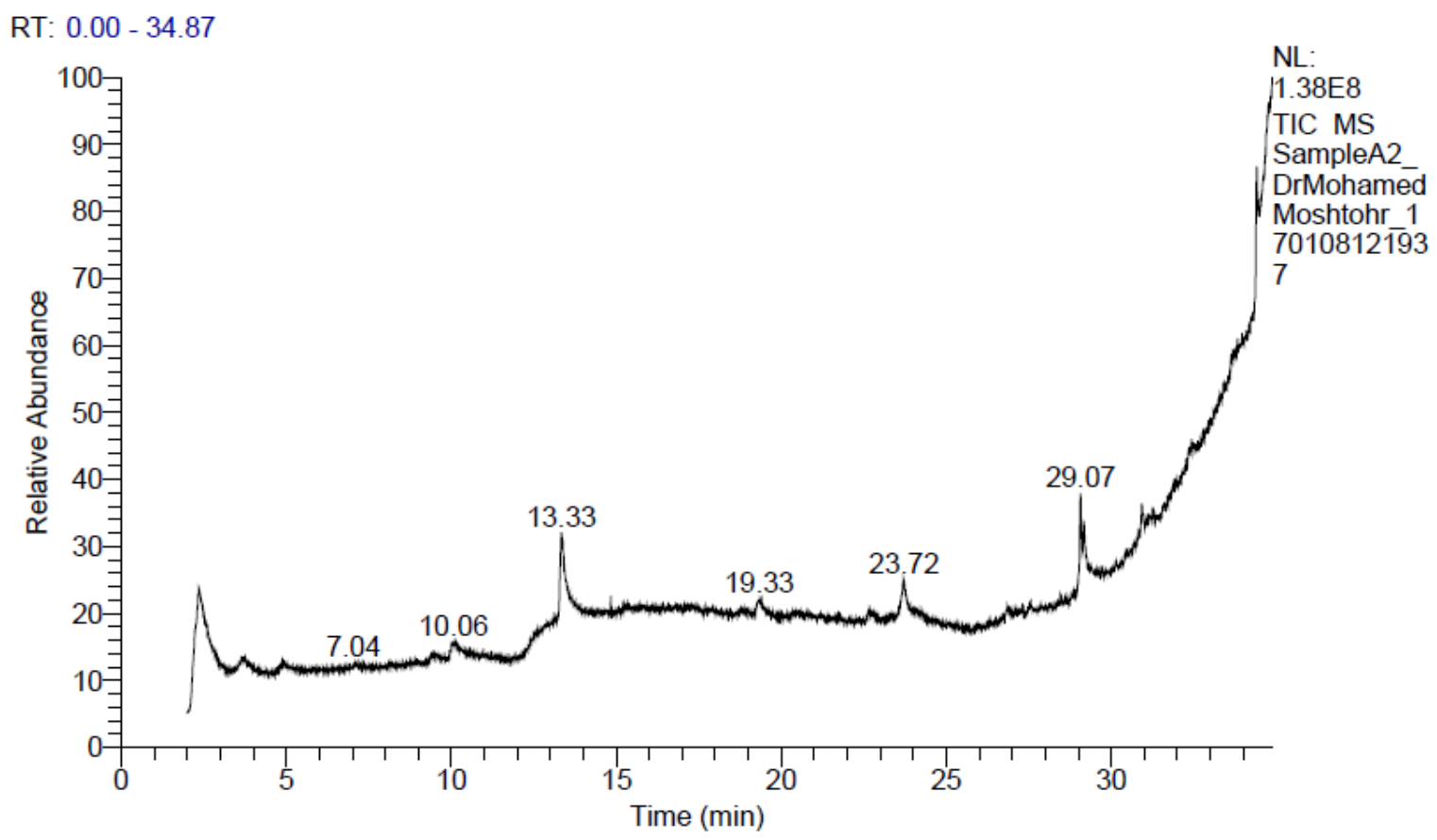

Fig 2. Identification and determination of apricot juice by GC/ MS chromatography analysis

Table 3. Identification and determination of orange juice by GC/ MS chromatography analysis:

\begin{tabular}{|c|c|c|c|c|c|}
\hline $\begin{array}{l}\text { Peak } \\
\text { No. }\end{array}$ & R.t* & Compound name & $\begin{array}{c}\text { Area } \\
\%\end{array}$ & $\begin{array}{l}\text { Molecular } \\
\text { Weight }\end{array}$ & $\begin{array}{l}\text { Molecular } \\
\text { formula }\end{array}$ \\
\hline 1 & 2.05 & Ethane, 1,1,2-trimethoxy- & 0.01 & 120 & $\mathrm{C} 5 \mathrm{H} 12 \mathrm{O} 3$ \\
\hline 2 & 2.18 & ETHANE, 1,1'-OXYBIS- & 79.36 & 74 & $\mathrm{C} 4 \mathrm{H} 10 \mathrm{O}$ \\
\hline 3 & 29.07 & 7-NONENOIC-7,8-D2 ACID, METHYL ESTER & 0.42 & 172 & $\mathrm{C} 10 \mathrm{H} 16 \mathrm{D} 2 \mathrm{O} 2$ \\
\hline 4 & 29.07 & 10-UNDECENOIC ACID, METHYL ESTER & 0.50 & 198 & $\mathrm{C} 12 \mathrm{H} 22 \mathrm{O} 2$ \\
\hline 5 & 29.18 & 13,16 -Octadecadiynoic acid, methyl ester & 0.80 & 290 & $\mathrm{C} 19 \mathrm{H} 30 \mathrm{O} 2$ \\
\hline 6 & 29.86 & 13-TETRADECYNOIC ACID, METHYL ESTER & 0.19 & 238 & $\mathrm{C} 15 \mathrm{H} 26 \mathrm{O} 2$ \\
\hline 7 & 30.83 & 14-PENTADECYNOIC ACID, METHYL ESTER & 0.46 & 252 & $\mathrm{C} 16 \mathrm{H} 28 \mathrm{O} 2$ \\
\hline 8 & 34.11 & $\begin{array}{l}\text { [1,1'-Bicyclopropyl]-2-octanoic acid, 2'-hexyl-, } \\
\text { methyl ester }\end{array}$ & 7.31 & 322 & $\mathrm{C} 21 \mathrm{H} 38 \mathrm{O} 2$ \\
\hline 9 & 34.41 & 9-OCTADECENOIC ACID (Z) & 16.94 & 282 & $\mathrm{C} 18 \mathrm{H} 34 \mathrm{O} 2$ \\
\hline 10 & 34.41 & $\begin{array}{l}\text { HEXADECANOIC ACID, } 2,3- \\
\text { DIHYDROXYPROPYL ESTER }\end{array}$ & 3.89 & 330 & C19H38O4 \\
\hline 11 & 35.18 & 9-Hexadecenoic acid & 1.52 & 254 & $\mathrm{C} 16 \mathrm{H} 30 \mathrm{O} 2$ \\
\hline 12 & 35.72 & $\begin{array}{l}\text { 9,10,11-OCTADECENOIC ACID (Z)-, METHYL } \\
\text { ESTER }\end{array}$ & 5.63 & 296 & $\mathrm{C} 19 \mathrm{H} 36 \mathrm{O} 2$ \\
\hline 13 & 36.46 & Oleic Acid & 12.01 & 282 & C18H34O2 \\
\hline 14 & 36.81 & $\begin{array}{l}\text { 2-HYDROXY-3-[(9E)-9-OCTAD } \\
\text { ECENOYLOXY]PROPYL (9E)-9 } \\
\text { OCTADECENOATE }\end{array}$ & 1.9 & 620 & C39H72O5 \\
\hline 15 & 38.09 & $\begin{array}{l}\text { 9-Octadecenoic acid, (2-phenyl-1,3-dioxolan-4- } \\
\text { yl)methyl ester, cis }\end{array}$ & 0.22 & 444 & $\mathrm{C} 28 \mathrm{H} 44 \mathrm{O} 4$ \\
\hline
\end{tabular}




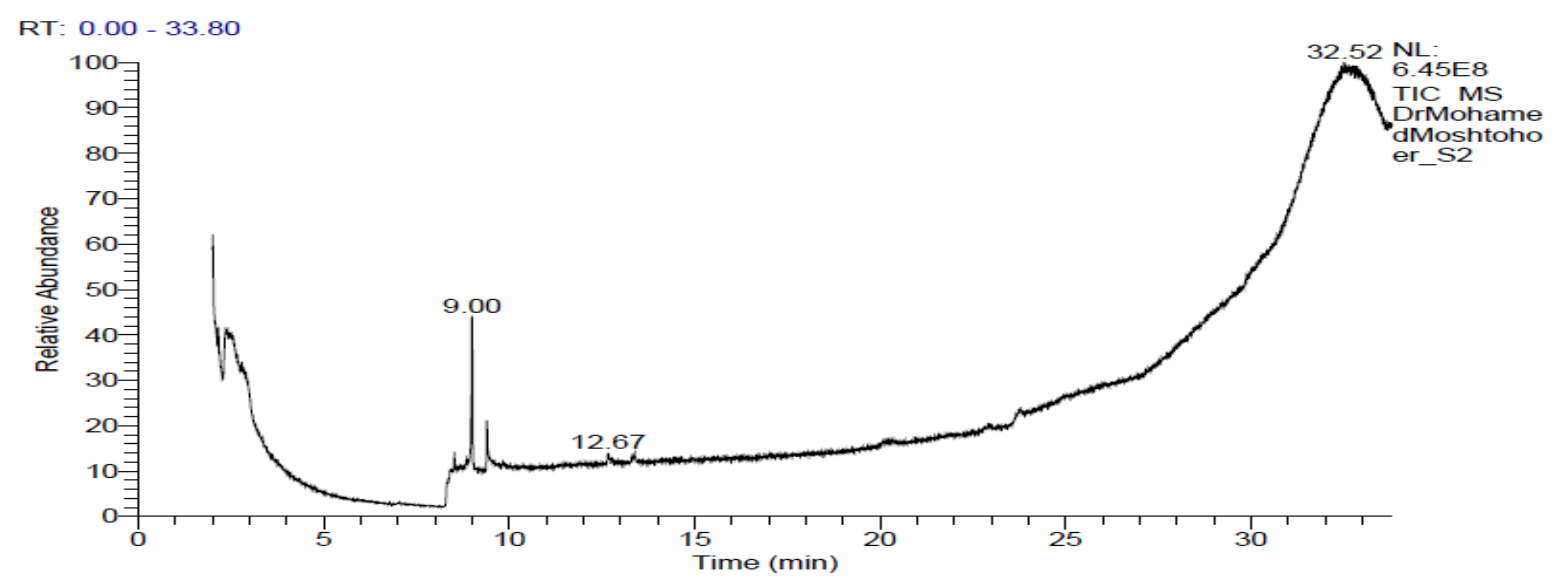

Fig 3. Identification and determination of orange juice by GC/ MS chromatography analysis

Data presented in Table (4) showed that determination of kaki juice by $\mathrm{GC} / \mathrm{MS}$ chromatography analysis compounds, illustrates that fractions were identified and could be related mainly to ketones, alcohols, fatty acids and hydrocarbons. Ketones and alcohols were the most predominate chemical fractions as ethane, 1,1'-oxybis ( 84.39), 9octadecenoic acid (z)-( 13.01), oleic acid (6.43), 9hexadecenoic acid (6.16) and 1heptatriacotanol(6.06). On the other hand, permethylated and reduced product of degradation product from h3-glycolipid by l-1-fucosidase and by b- galactosidase (0.06) was the lowest contents compounds.

Results on (Table 4) were in agreement with those reported by Zhu, et al (2016) found that AcW strongly affected the sensory qualities and the volatile compounds of persimmon wine. Compared with PW, AcW significantly improved the flavour of persimmon wine, which was attributed to the fact that AcW was capable of extracting more amino acids from the persimmon fruit than PW or AlW. In addition, these amino acids were converted into volatiles, mainly alcohols and esters, that affected the flavour of the resulting persimmon wine .

Table 4. Identification and determination of kaki juice by GC/ MS chromatography analysis:

\begin{tabular}{|c|c|c|c|c|c|}
\hline $\begin{array}{l}\text { Peak } \\
\text { No. }\end{array}$ & R.t* & Compound name & $\begin{array}{c}\text { Area } \\
\%\end{array}$ & $\begin{array}{l}\text { Molecular } \\
\text { Weight }\end{array}$ & $\begin{array}{l}\text { Molecular } \\
\text { formula }\end{array}$ \\
\hline 1 & 2.46 & ETHANE, 1,1'-OXYBIS & 84.39 & 74 & $\mathrm{C} 4 \mathrm{H} 10 \mathrm{O}$ \\
\hline 2 & 3.43 & $\begin{array}{l}\text { [1,1'-Bicyclopropyl]-2-octanoic acid, 2'-hexyl- } \\
\text {, methyl ester }\end{array}$ & 0.11 & 322 & $\mathrm{C} 21 \mathrm{H} 38 \mathrm{O} 2$ \\
\hline 3 & 3.43 & $\begin{array}{l}\text { PERMETHYLATED AND REDUCED } \\
\text { PRODUCT OF DEGRADATION PRODUCT } \\
\text { FROM H3-GLYCOLIPID BY L-L- } \\
\text { FUCOSIDASE AND BY B- } \\
\text { GALACTOSIDASE }\end{array}$ & 0.06 & 1780 & C94H180N4O26 \\
\hline 4 & 3.43 & $\begin{array}{l}\text { 9,10-SECOCHOLA-5,7,10(19)-T RIENE- } \\
\text { 3,24-DIOL, (3á,5Z,7E) }\end{array}$ & 0.14 & 358 & $\mathrm{C} 24 \mathrm{H} 38 \mathrm{O} 2$ \\
\hline 5 & 13.07 & Dodecanoic acid, 3-hydroxy & 0.30 & 216 & $\mathrm{C} 12 \mathrm{H} 24 \mathrm{O} 3$ \\
\hline 6 & 26.97 & $\begin{array}{l}\text { 11,14,13,16-Octadecadiynoic acid, methyl } \\
\text { ester }\end{array}$ & 0.42 & 290 & $\mathrm{C} 19 \mathrm{H} 30 \mathrm{O} 2$ \\
\hline 7 & 34.03 & ALANINE, 3-(BENZYLOXY)-, L- & 2.56 & 195 & $\mathrm{C} 10 \mathrm{H} 13 \mathrm{NO} 3$ \\
\hline 8 & 34.03 & 9-OCTADECENOIC ACID (Z)- & 13.01 & 282 & $\mathrm{C} 18 \mathrm{H} 34 \mathrm{O} 2$ \\
\hline 9 & 34.03 & Oleic Acid & 6.43 & 282 & $\mathrm{C} 18 \mathrm{H} 34 \mathrm{O} 2$ \\
\hline 10 & 34.03 & $\begin{array}{l}\text { 2-HYDROXY-3-[(9E)-9-OCTAD } \\
\text { ECENOYLOXY]PROPYL }\end{array}$ & 4.19 & 620 & $\mathrm{C} 39 \mathrm{H} 72 \mathrm{O} 5$ \\
\hline 11 & 34.60 & 1-Heptatriacotanol & 6.06 & 536 & $\mathrm{C} 37 \mathrm{H} 76 \mathrm{O}$ \\
\hline 12 & 36.55 & Ethyl iso-allocholate & 1.83 & 436 & $\mathrm{C} 26 \mathrm{H} 44 \mathrm{O} 5$ \\
\hline 13 & 36.74 & 9-Hexadecenoic acid & 6.16 & 254 & $\mathrm{C} 16 \mathrm{H} 30 \mathrm{O} 2$ \\
\hline
\end{tabular}

*R.t, retention time $(\mathrm{min})$.

(values expressed as relative area percentage to total identified compounds). 


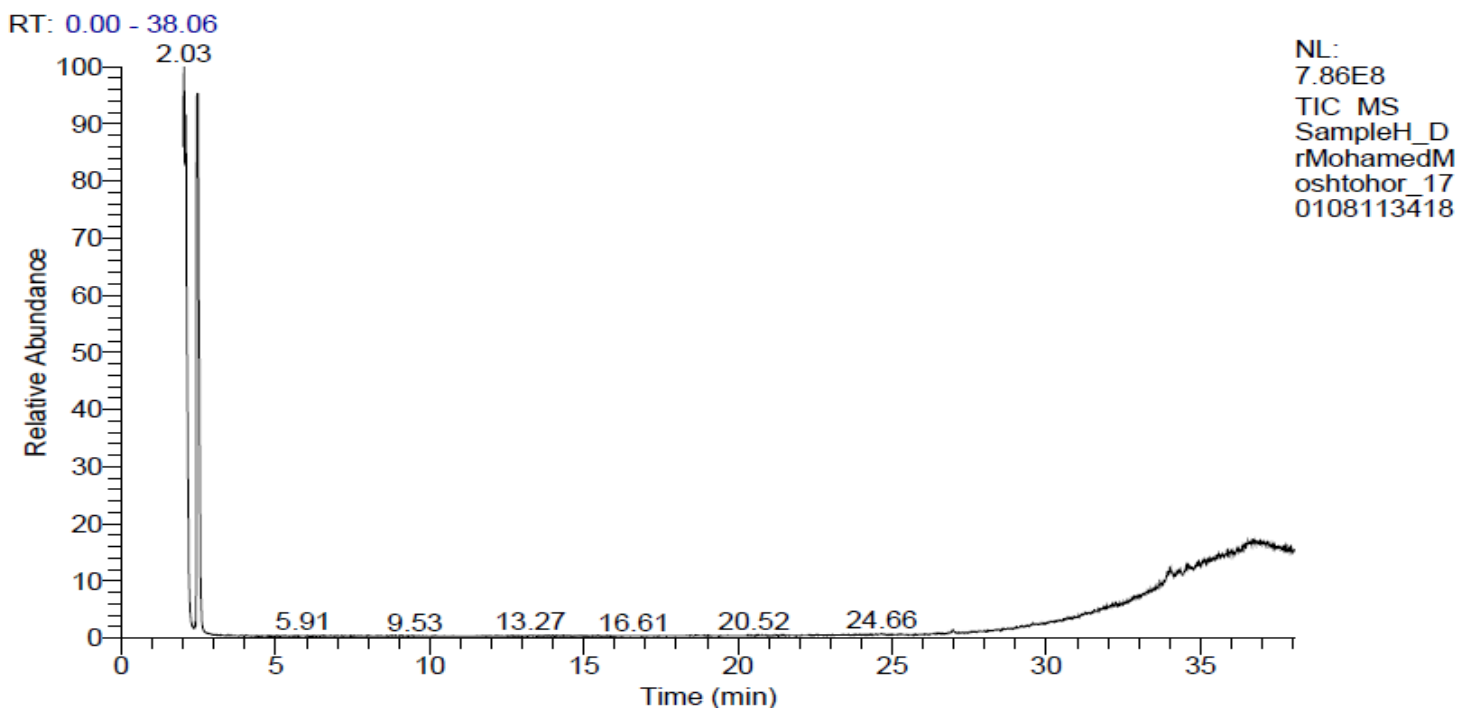

Fig 4. Identification and determination of kaki juice by GC/ MS chromatography analysis

Data presented in Table (5) showed that determination of carrot juice by $\mathrm{GC} / \mathrm{MS}$ chromatography analysis compounds, which could be classified mainly as aldehyde ketones, alcohols, fatty acids and hydrocarbons. Aldehydes and alcohols were the most representative chemical fractions and contents were the highest 9-octadecenoic acid (z)(40.31), oleic acid (20.55) and 9-octadecenoic acid (z)-,methyl ester (10.74). on the other hand, ethane, 1,1'-oxybis-( 2.94), 1-ethoxyethane (3.04) and isochiapin $b$ ( 5.12) were the lowest contents compounds.
Results on (Table 5) were in agreement with those reported by Fukuda,et al (2016) found that the aroma attribute intensity and volatile contents of the Kuroda type was lower than those of other carrot types. Therefore, using the Kuroda types to breed new carrot varieties will reduce the content of certain chemical components, and thus, the F1 hybrid carrots will have less harshness and bitterness. Abu el- maaty (2012) found that, carrot juice 1- Penten-3-one $15.21 \%$ - 1- Penten-3- ol 14.06\%- Methyl butanoate $13.42 \%$ was less concentration Methannol $0.02 \%$.

Table 5. Identification and determination of carrot juice by GC/ MS chromatography analysis:

\begin{tabular}{|c|c|c|c|c|c|}
\hline $\begin{array}{l}\text { Peak } \\
\text { No. }\end{array}$ & R.t* & Compound name & Area \% & $\begin{array}{l}\text { Molecular } \\
\text { Weight }\end{array}$ & $\begin{array}{l}\text { Molecular } \\
\text { formula }\end{array}$ \\
\hline 1 & 2.04 & 1-ETHOXYETHANE & 3.04 & 74 & $\mathrm{C} 4 \mathrm{H} 10 \mathrm{O}$ \\
\hline 2 & 4.18 & ETHANE, 1,1'-OXYBIS- & 2.94 & 74 & $\mathrm{C} 4 \mathrm{H} 10 \mathrm{O}$ \\
\hline 3 & 12.90 & Dodecanoic acid, 3-hydroxy- & 8.22 & 216 & $\mathrm{C} 12 \mathrm{H} 24 \mathrm{O} 3$ \\
\hline 4 & 25.37 & 9-Hexadecenoic acid & 7.97 & 254 & $\mathrm{C} 16 \mathrm{H} 30 \mathrm{O} 2$ \\
\hline 5 & 34.43 & Oleic Acid & 20.55 & 282 & $\mathrm{C} 18 \mathrm{H} 34 \mathrm{O} 2$ \\
\hline 6 & 35.71 & $\begin{array}{l}\text { 9-OCTADECENOIC ACID (Z)-,METHYL } \\
\text { ESTER }\end{array}$ & 10.74 & 296 & $\mathrm{C} 19 \mathrm{H} 36 \mathrm{O} 2$ \\
\hline 7 & 36.50 & 9-OCTADECENOIC ACID (Z)- & 40.31 & 282 & C18H34O2 \\
\hline 8 & 38.59 & ISOCHIAPIN B & 5.12 & 346 & $\mathrm{C} 19 \mathrm{H} 22 \mathrm{O} 6$ \\
\hline 9 & 2.04 & 1-ETHOXYETHANE & 3.04 & 74 & $\mathrm{C} 4 \mathrm{H} 10 \mathrm{O}$ \\
\hline
\end{tabular}

*R.t, retention time ( $\mathrm{min})$.

(values expressed as relative area percentage to total identified compounds). 


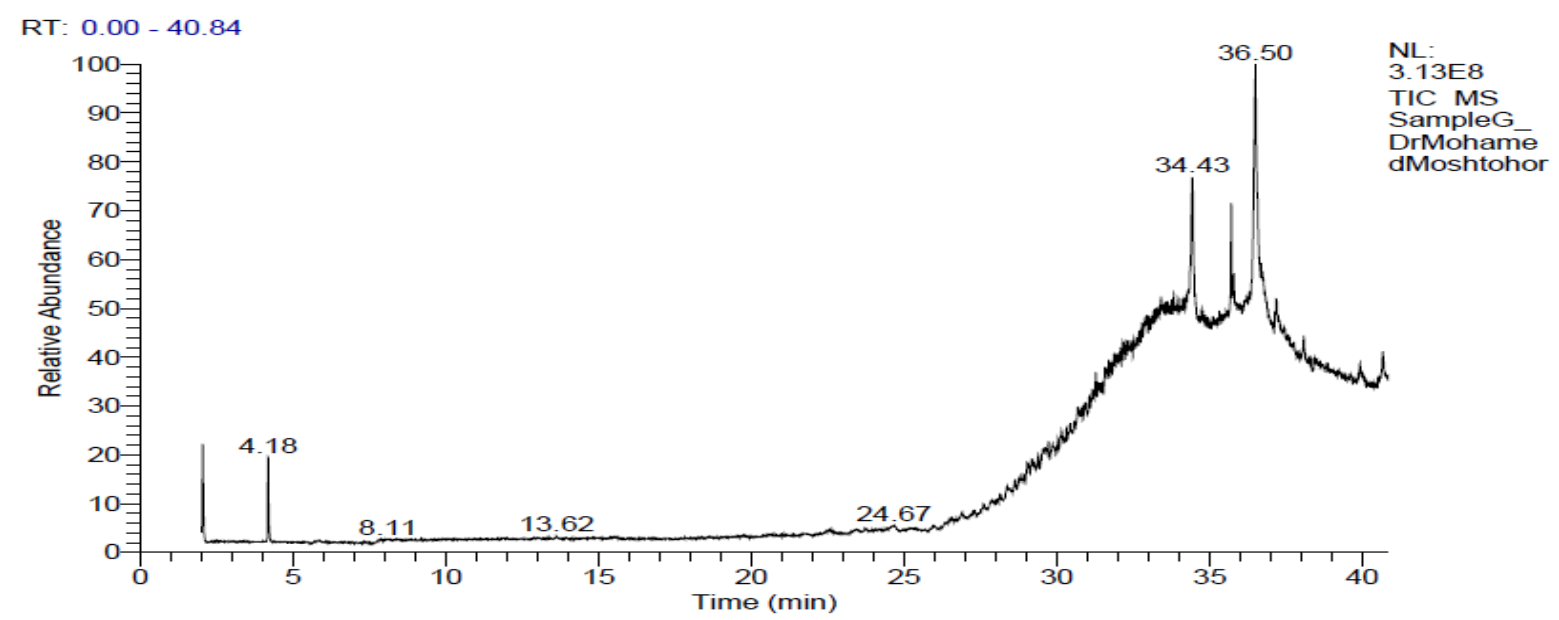

Fig 5. Identification and determination of carrot juice by GC/ MS chromatography analysis

Data presented in Table (6) illustrated GC/MS analysis of fresh mango juice treated with addition bectinase enzyme. Ten compounds mainly the identified fractions were related to: ketones, alcohols, fatty acids and hydrocarbons, where ketones and alcohols were the most representative chemical fraction as 9-octadecenoic acid (z)-( 86.88), 9hexadecenoic acid( 76.41) and oleic acid (76.27) has been found as highest fractions. In addition to the lowest contents compounds volatiles fractions in (Table 6) was Dodecanoic acid, 3-hydroxy-(2.73) .

Results on (Table 6) were enzymatic pretreatment of chopped fruit materials may hydrolyze galacturonic acid and polysaccharides to check titrable acidity (TA) and total soluble solids (TSS), (Sharma et al., 2017) and Chauhan, et.al (2001).

Table 6. Volatile compounds identified in headspace of fresh mango juice treated with addition bectinase enzyme by GC/ MS chromatography analysis.

\begin{tabular}{|c|c|c|c|c|c|}
\hline $\begin{array}{l}\text { Peak } \\
\text { No. }\end{array}$ & R.t* & Compound name & $\underset{\%}{\text { Area }}$ & $\begin{array}{l}\text { Molecular } \\
\text { Weight }\end{array}$ & $\begin{array}{l}\text { Molecular } \\
\text { formula }\end{array}$ \\
\hline 1 & 2.71 & $\begin{array}{l}{[1,1 \text { '-Bicyclopropyl }]-2 \text {-octanoic acid, 2'-hexyl-, }} \\
\text { methyl ester }\end{array}$ & 6.64 & 322 & $\mathrm{C} 21 \mathrm{H} 38 \mathrm{O} 2$ \\
\hline 2 & 2.71 & $\begin{array}{l}\text { PERMETHYLATED AND REDUCED } \\
\text { PRODUCT OF DEGRADATION } \\
\text { PRODUCT FROM H3-GLYCOLIPID BY L-L- } \\
\text { FUCOSIDASE AND BY B GALACTOSIDASE }\end{array}$ & 5.97 & 1780 & C94H180N4O2 \\
\hline 3 & 2.85 & 13,16-Octadecadiynoic acid, methyl ester & 9.32 & 290 & $\mathrm{C} 19 \mathrm{H} 30 \mathrm{O} 2$ \\
\hline 4 & 23.88 & 1,5,5-Trimethyl-6-methylene-cyclo hexane & 3.43 & 136 & $\mathrm{C} 10 \mathrm{H} 16$ \\
\hline 5 & 33.23 & Dodecanoic acid, 3-hydroxy- & 2.73 & 216 & $\mathrm{C} 12 \mathrm{H} 24 \mathrm{O} 3$ \\
\hline 6 & 33.23 & $\begin{array}{l}\text { 2-AMINOETHANETHIOL HYDROGEN } \\
\text { SULFATE (ESTER) }\end{array}$ & 4.34 & 157 & $\mathrm{C} 2 \mathrm{H} 7 \mathrm{NO} 3 \mathrm{~S} 2$ \\
\hline 7 & 34.27 & ALANINE, 3-(BENZYLOXY)-, L- & 3.17 & 195 & $\mathrm{C} 10 \mathrm{H} 13 \mathrm{NO} 3$ \\
\hline 8 & 38.84 & 9-OCTADECENOIC ACID (Z)- & 86.88 & 282 & $\mathrm{C} 18 \mathrm{H} 34 \mathrm{O} 2$ \\
\hline 9 & 39.21 & 9-Hexadecenoic acid & 76.41 & 254 & $\mathrm{C} 16 \mathrm{H} 30 \mathrm{O} 2$ \\
\hline 10 & 39.21 & Oleic Acid & 76.27 & 282 & $\mathrm{C} 18 \mathrm{H} 34 \mathrm{O} 2$ \\
\hline
\end{tabular}

*R.t, retention time (min).

(values expressed as relative area percentage to total identified compounds). 


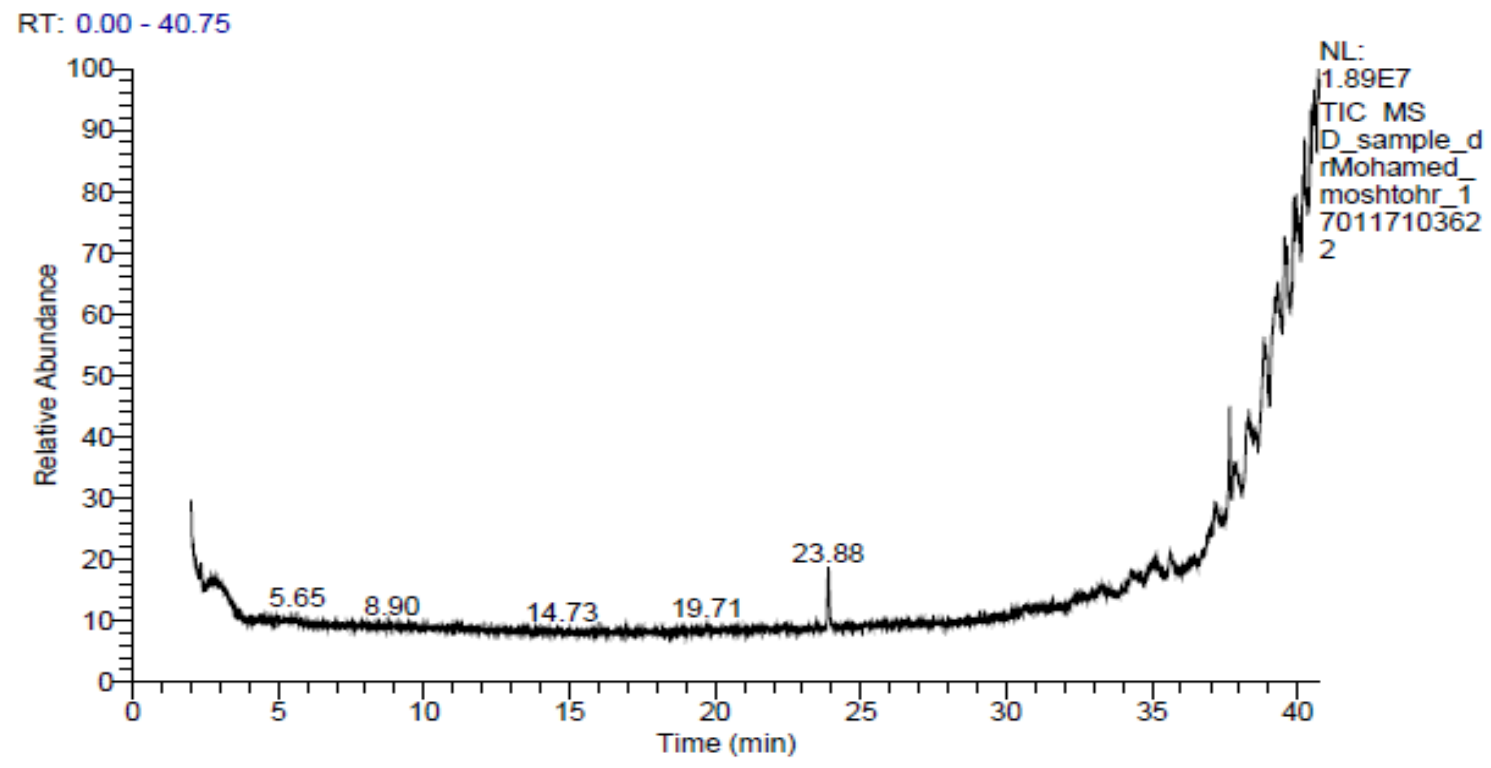

Fig(6): Volatile compounds identified in headspace of fresh mango juice treated with addition bectinase enzyme by GC/ MS chromatography analysis

Data presented in Table (7) showed the results of the GC-MS analysis of the volatiles isolated from fresh mango juice treated with addition cellulase enzyme. Table (7) illustrates that fractions were identified and could be related mainly to ketones, alcohols, fatty acids and hydrocarbons. Ketones and alcohols were the most predominate chemical fractions as 9-octadecenoic acid (z) (96.29), oleic acid
(79.73) and hexadecanoic acid (14.01) has been found as highest contents compounds volatiles fractions. in addition to the lowest contents compounds volatiles fractions in (Table 7) was 9-octadecenoic acid, (2phenyl-1,3-dioxolan-4-yl)methyl ester, cis- ( 2.35) . Results on (Table 7) were color changed with oxidation, not with enzyme-assisted treatment and storage time, Mihalev et al. (2004).

Table 7. Volatile compounds identified in headspace of fresh mango juice treated with addition cellulase enzyme by GC/ MS chromatography analysis:

\begin{tabular}{|c|c|c|c|c|c|}
\hline $\begin{array}{c}\text { Peak } \\
\text { No. }\end{array}$ & R.t* & Compound name & $\begin{array}{c}\text { Area } \\
\%\end{array}$ & $\begin{array}{c}\text { Molecular } \\
\text { Weight }\end{array}$ & $\begin{array}{c}\text { Molecular } \\
\text { formula }\end{array}$ \\
\hline 1 & 29.46 & HEXADECANOIC ACID & 14.01 & 256 & $\mathrm{C} 16 \mathrm{H} 32 \mathrm{O} 2$ \\
\hline 2 & 30.72 & 9,10-Octadecenoic acid, methyl ester & 4.5 & 296 & $\mathrm{C} 19 \mathrm{H} 36 \mathrm{O} 2$ \\
\hline 3 & 31.41 & 9-OCTADECENOIC ACID (Z) & 96.29 & 282 & $\mathrm{C} 18 \mathrm{H} 34 \mathrm{O} 2$ \\
\hline 4 & 31.41 & Oleic Acid & 79.73 & 282 & $\mathrm{C} 18 \mathrm{H} 34 \mathrm{O} 2$ \\
\hline 5 & 32.67 & 1-Heptatriacotanol & 3.99 & 536 & $\mathrm{C} 37 \mathrm{H} 76 \mathrm{O}$ \\
\hline 6 & 34.68 & 9-Octadecenoic acid, (2-phenyl-1,3-dioxolan-4- & 2.35 & 444 & $\mathrm{C} 28 \mathrm{H} 44 \mathrm{O} 4$ \\
\hline
\end{tabular}

*R.t, retention time $(\mathrm{min})$.

(values expressed as relative area percentage to total identified compounds). 


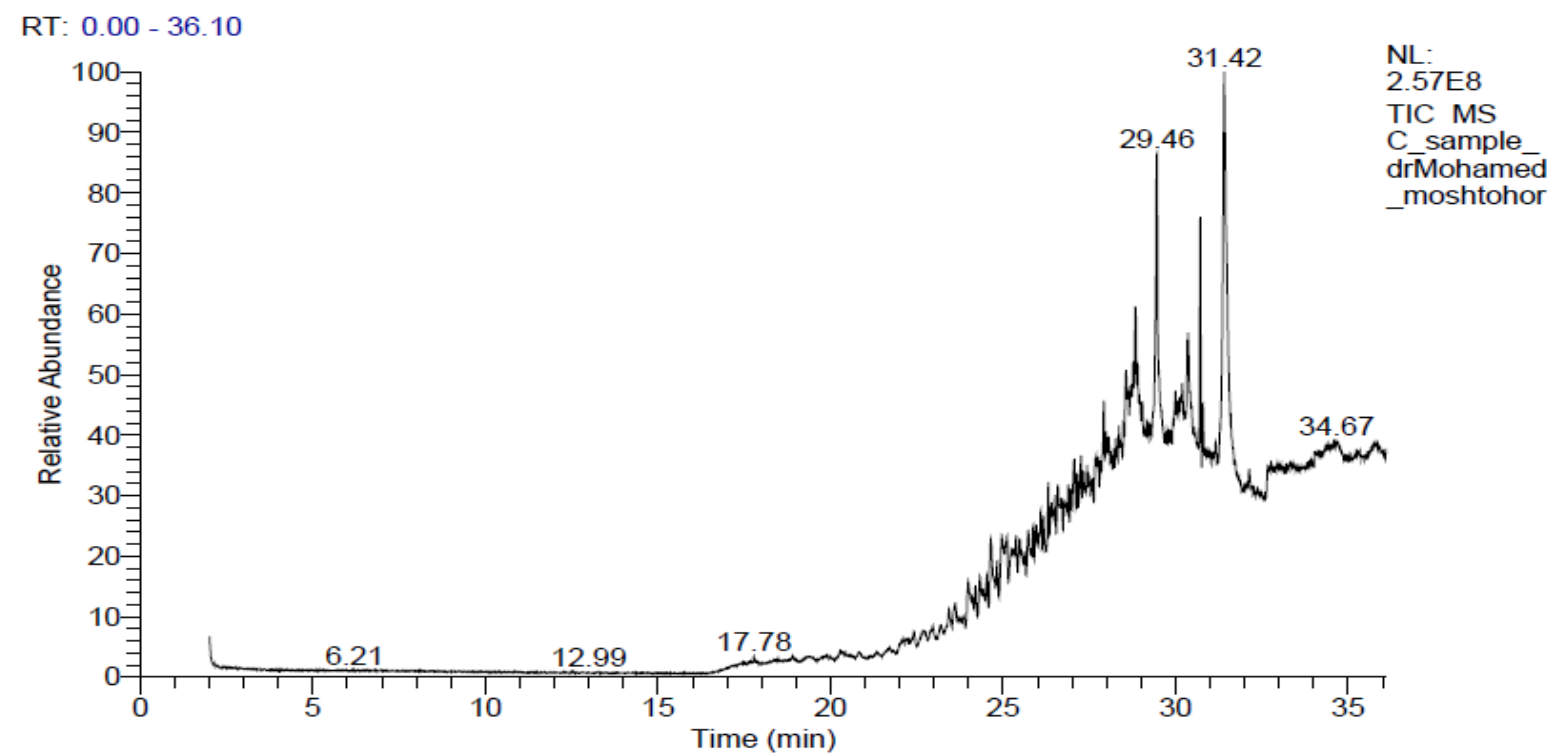

Fig 7. Volatile compounds identified in headspace of fresh mango juice treated with addition cellulase enzyme by GC/ MS chromatography analysis

Data presented in Table (8) showed that determination of fresh mango blending with orange juice by $\mathrm{GC} / \mathrm{MS}$ chromatography analysis compounds, which could be classified mainly as aldehyde ketones, alcohols, fatty acids and hydrocarbons. Aldehydes and alcohols were the most representative chemical fractions and contents were the highest 9-octadecenoic acid (z) ( 54.91), 10- octadecenoic acid, methyl ester (38.27), oleic acid (35.48) , ethane, 1,1'-oxybis- ( 32.7$)$ and [1,1'bicyclopropyl]-2-octanoic acid, 2'-hexyl-, methyl ester ( 32.33) . on the other hand, 2-hydroxy-3-[(9e)9-octad ecenoyloxy]propyl ( 8.90) and hexadecanoic acid, 2,3-dihydroxypropyl ester ( 11.69) were the lowest contents compounds.

Table 8. Volatile compounds identified in headspace of fresh mango blending with orange juice by GC/ MS chromatography analysis:

\begin{tabular}{|c|c|c|c|c|c|}
\hline $\begin{array}{l}\text { Peak } \\
\text { No. }\end{array}$ & R.t* & Compound name & $\begin{array}{c}\text { Area } \\
\%\end{array}$ & $\begin{array}{l}\text { Molecula } \\
\text { r Weight }\end{array}$ & $\begin{array}{c}\text { Molecular } \\
\text { formula }\end{array}$ \\
\hline 1 & 28.37 & ETHANE, 1,1'-OXYBIS- & 32.7 & 74 & $\mathrm{C} 4 \mathrm{H} 10 \mathrm{O}$ \\
\hline 2 & 29.26 & $\begin{array}{l}\text { [1,1'-Bicyclopropyl]-2-octanoic acid, 2'-hexyl-, } \\
\text { methyl ester }\end{array}$ & 32.33 & 322 & $\mathrm{C} 21 \mathrm{H} 38 \mathrm{O} 2$ \\
\hline 3 & 33.86 & 9-OCTADECENOIC ACID (Z) & 54.91 & 282 & $\mathrm{C} 18 \mathrm{H} 34 \mathrm{O} 2$ \\
\hline 4 & 33.86 & Oleic Acid & 35.48 & 282 & $\mathrm{C} 18 \mathrm{H} 34 \mathrm{O} 2$ \\
\hline 5 & 33.94 & $\begin{array}{l}\text { 2-HYDROXY-3-[(9E)-9-OCTAD } \\
\text { ECENOYLOXY]PROPYL }\end{array}$ & 8.90 & 620 & C39H72O5 \\
\hline 6 & 33.94 & $\begin{array}{l}\text { HEXADECANOIC ACID, } 2,3- \\
\text { DIHYDROXYPROPYL ESTER }\end{array}$ & 11.69 & 330 & C19H38O4 \\
\hline 7 & 36.34 & 10-Octadecenoic acid, methyl ester & 38.27 & 296 & $\mathrm{C} 19 \mathrm{H} 36 \mathrm{O} 2$ \\
\hline
\end{tabular}

*R.t, retention time (min).

(values expressed as relative area percentage to total identified compounds). 


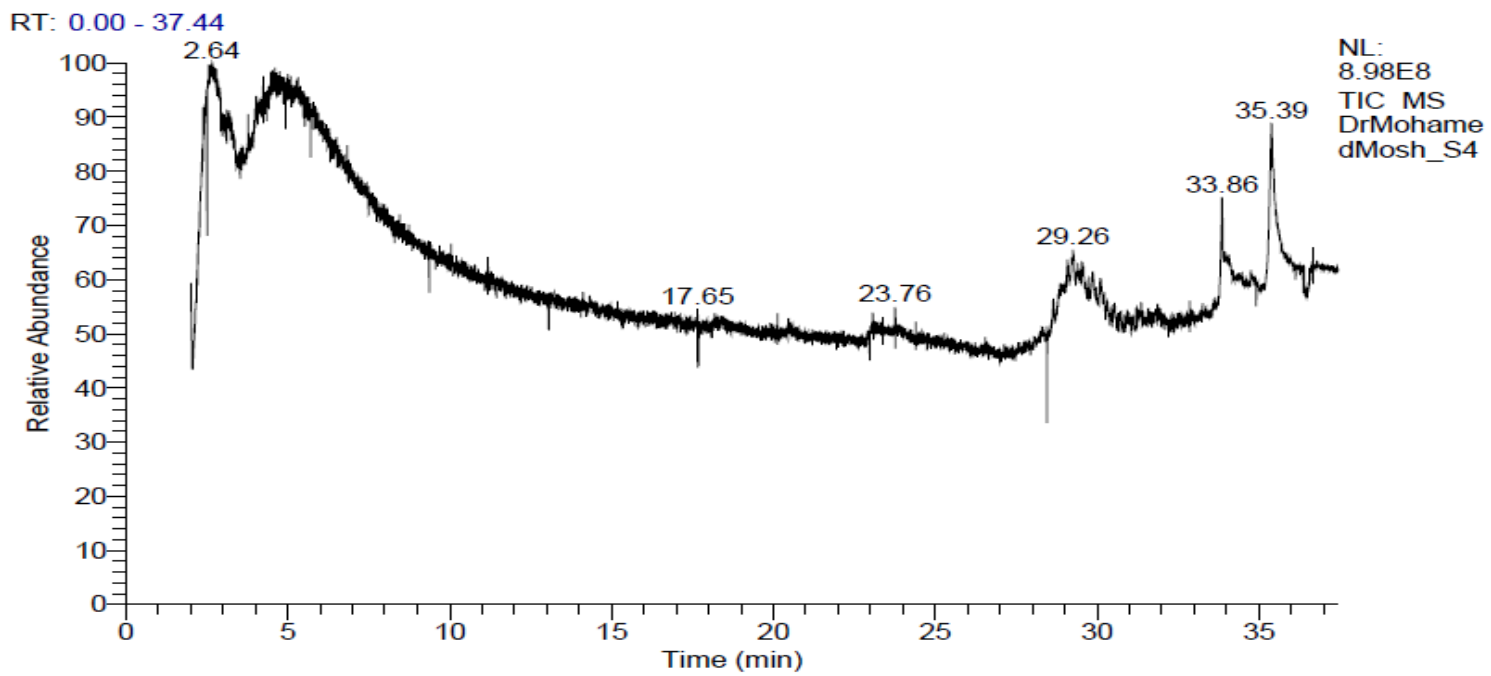

Fig 8. Volatile compounds identified in headspace of fresh mango blending with orange juice by GC/ MS chromatography analysis

Data presented in Table (9) showed that determination of fresh mango blending with carrot juice by $\mathrm{GC} / \mathrm{MS}$ chromatography analysis compounds, which could be classified mainly as aldehyde ketones, alcohols, fatty acids and hydrocarbons. Aldehydes and alcohols were the most representative chemical fractions and contents was the highest ethane, 1,1'-oxybis (70.35) . on the other hand, 2,2-dideutero octadecanal (4.06), 7-nonenoic-7,8-d2 acid,methyl ester ( 4.59) , 7-nonenoic-7,8-d2 acid,methyl ester (4.59), 8-nonenoic-8,9-d2 acid,methyl ester (4.59), 10-undecenoic acid,methyl ester(4.59)and permethylated and reduced product of degradation product from h3-glycolipid by 1-1fucosidase and by b-galactosidase ( 4.59) were the lowest contents compounds.

Results on (Table 9) were in agreement with those reported by Abu el- maaty (2012) found that, the compound Ethyl isobutyrate $20.96 \%-11.22 \%$ in nectar papaya apricot and papaya carrot. The lowest concentration was compound Ethyl decanoate $0.01 \%$ in nectar papaya, apricot and compound limonene $0.06 \%$ nectar papaya apricot.

Table 9. Volatile compounds identified in headspace of fresh mango blending with carrot juice by GC/ MS chromatography analysis:

\begin{tabular}{ccl}
$\begin{array}{c}\text { Peak } \\
\text { No. }\end{array}$ & R.t* & \multicolumn{1}{c}{ Compound name } \\
1 & 9.00 & ETHANE, 1,1'-OXYBIS \\
2 & 23.77 & 7-NONENOIC-7,8-D2 ACID,METHYL ESTER \\
3 & 23.77 & 8-NONENOIC-8,9-D2 ACID,METHYL ESTER \\
4 & 23.77 & 10-UNDECENOIC ACID,METHYL ESTER \\
5 & 23.77 & PERMETHYLATED AND REDUCED \\
& & PRODUCT OF DEGRADATION PRODUCT \\
& & FROM H3-GLYCOLIPID BY L-L-FUCOSIDASE \\
& & AND BY B-GALACTOSIDASE \\
6 & 32.52 & Z-(13,14-Epoxy)tetradec-11-en-1-olacetate \\
7 & 32.52 & Z-8-Methyl-9-tetradecenoic acid E-8-Methyl-7- \\
& & dodecen-1-ol acetate \\
8 & 32.52 & 6-Acetyl-á-d-mannose \\
9 & 32.97 & 1-Dodecanol, 3,7,11-trimethyl- \\
10 & 33.32 & 2,2-DIDEUTERO OCTADECANAL
\end{tabular}

\begin{tabular}{ccl}
$\begin{array}{c}\text { Area } \\
\%\end{array}$ & $\begin{array}{c}\text { Molecular } \\
\text { Weight }\end{array}$ & \multicolumn{1}{c}{$\begin{array}{c}\text { Molecular } \\
\text { formula }\end{array}$} \\
70.35 & 74 & C4H10O \\
4.59 & 172 & C10H16D2O2 \\
4.59 & 172 & $\mathrm{C} 10 \mathrm{H} 16 \mathrm{D} 2 \mathrm{O} 2$ \\
4.59 & 198 & $\mathrm{C} 12 \mathrm{H} 22 \mathrm{O} 2$ \\
4.59 & 1780 & $\mathrm{C} 94 \mathrm{H} 180 \mathrm{~N} 4 \mathrm{O} 26$
\end{tabular}

$*$ R.t, retention time (min).

(values expressed as relative area percentage to total identified compounds). 


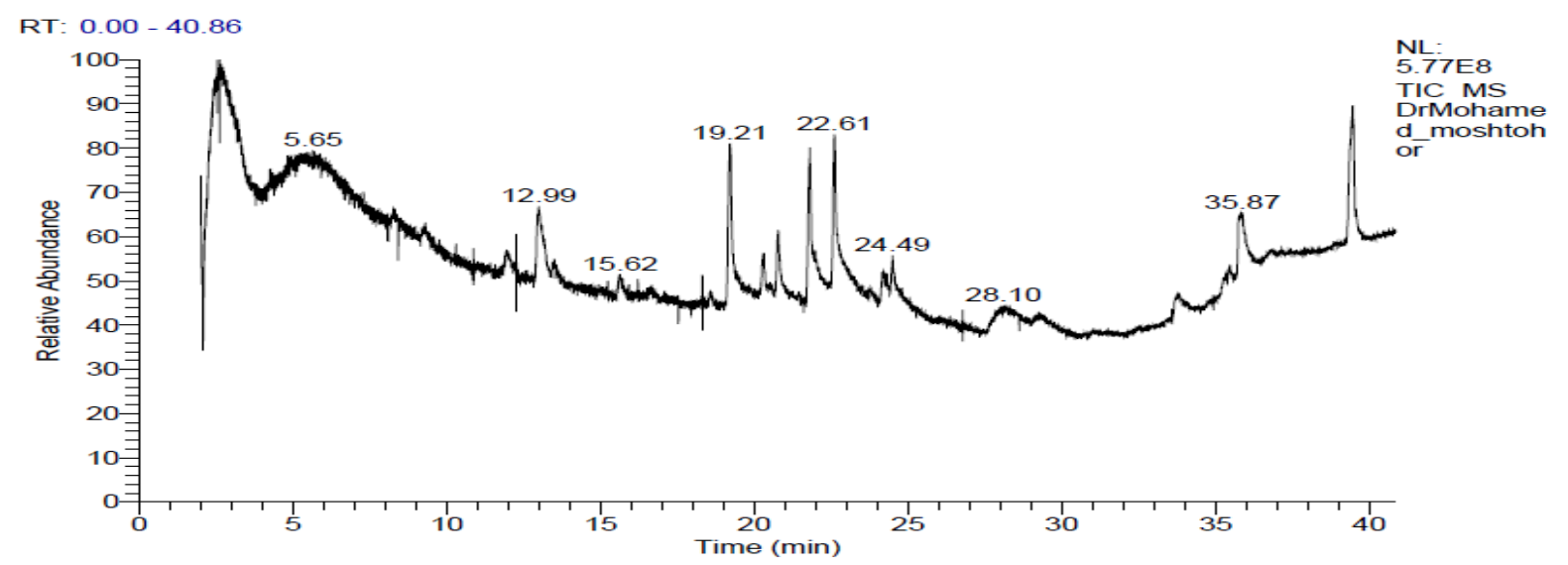

Fig(9): Volatile compounds identified in headspace of fresh mango blending with carrot juice by GC/ MS chromatography analysis

Data presented in Table (10) illustrated GC/MS analysis of fresh mango blending with kika juice. Nine compounds mainly the identified fractions were related to: ketones, alcohols, fatty acids and hydrocarbons, where ketones and alcohols were the most representative chemical fraction as ethane, 1,1'-oxybis (89.07), 2-butanol, 1-methoxy (78.85) and ethane, 1,2-diethoxy(78.85) has been found as highest fractions. In addition to the lowest contents compounds volatiles fractions in (Table 10) was 13Tetradecynoic acid, methyl ester (0.76) .

Table 10. Volatile compounds identified in headspace of fresh mango blending with kika juice by GC/ MS chromatography analysis:

\begin{tabular}{|c|c|c|c|c|c|}
\hline $\begin{array}{l}\text { Peak } \\
\text { No. }\end{array}$ & R.t* & Compound name & $\underset{\%}{\text { Area }}$ & $\begin{array}{l}\text { Molecular } \\
\text { Weight }\end{array}$ & $\begin{array}{l}\text { Molecular } \\
\text { formula }\end{array}$ \\
\hline 1 & 2.14 & ETHANE, 1,1'-OXYBIS & 89.07 & 74 & $\mathrm{C} 4 \mathrm{H} 10 \mathrm{O}$ \\
\hline 2 & 2.14 & 2-Butanol, 1-methoxy & 78.85 & 104 & $\mathrm{C} 5 \mathrm{H} 12 \mathrm{O} 2$ \\
\hline 3 & 2.14 & Ethane, 1,2-diethoxy & 78.85 & 118 & C6H14O2 \\
\hline 4 & 30.08 & 13-Tetradecynoic acid, methyl ester & 0.76 & 238 & C15H26O2 \\
\hline 5 & 31.27 & $\begin{array}{l}\text { [1,1'-Bicyclopropyl]-2-octanoic acid, 2'-hexyl-, } \\
\text { methyl ester }\end{array}$ & 3.74 & 322 & $\mathrm{C} 21 \mathrm{H} 38 \mathrm{O} 2$ \\
\hline 6 & 31.27 & 9-Octadecenoic acid (Z)-, methyl ester & 2.33 & 296 & C19H36O2 \\
\hline 7 & 33.86 & Oleic Acid & 4.14 & 282 & C18H34O2 \\
\hline 8 & 33.86 & $\begin{array}{l}\text { HEXADECANOIC ACID, 2,3- } \\
\text { DIHYDROXYPROPYL ESTER }\end{array}$ & 3.12 & 330 & C19H38O4 \\
\hline 9 & 35.39 & OCTADEC-9-ENOIC ACID & 9.91 & 282 & $\mathrm{C} 18 \mathrm{H} 34 \mathrm{O} 2$ \\
\hline
\end{tabular}

*R.t, retention time $(\min )$.

(values expressed as relative area percentage to total identified compounds).

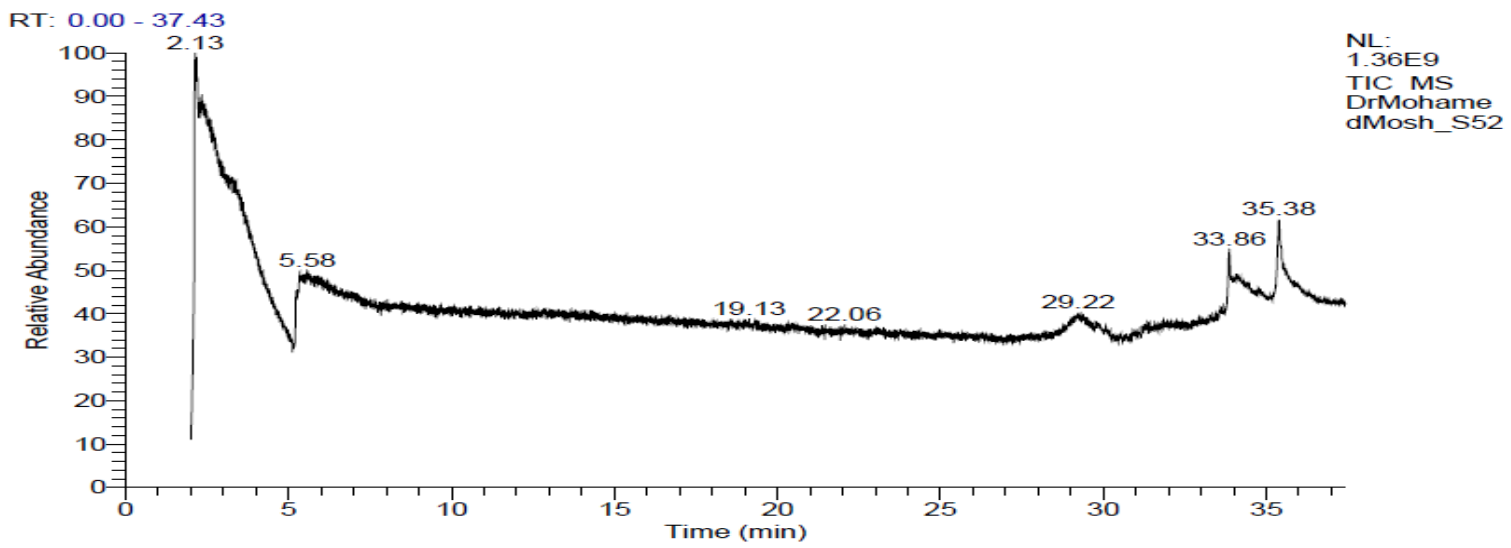

Fig(10): Volatile compounds identified in headspace of fresh mango blending with kika juice by GC/ MS chromatography analysis 
Data presented in Table (11) illustrated GC/MS analysis of fresh mango blending with apricot juice. Five compounds mainly the identified fractions were related to: ketones, alcohols, fatty acids and hydrocarbons, where ketones and alcohols were the most representative chemical fraction as 2-butanol, 1methoxy-( 30.18), oleic acid (29.15), ethane, 1,1,2trimethoxy- ( 19.02) and d-(+)-glyceric acid ( 15.57) has been found as highest fractions. In addition to the lowest contents compounds volatiles fractions in (Table 10) was 11-Octadecenoic acid, methyl ester (6.07).

Results on (Table 11) were in agreement with those reported by Abu el- maaty (2012) found that, Disappeared compounds $\alpha$ - terpineol, thyhexanoate, cymene and hexen-1-ol in nectar papaya apricot and papaya carrot, respectively immediately after pasteurization.

Table 11. Volatile compounds identified in headspace of fresh mango blending with apricot juice by GC/ MS chromatography analysis:

\begin{tabular}{cclccl}
\hline $\begin{array}{c}\text { Peak } \\
\text { No. }\end{array}$ & R.t* & \multicolumn{1}{c}{ Compound name } & $\begin{array}{c}\text { Area } \\
\mathbf{\%}\end{array}$ & $\begin{array}{c}\text { Molecular } \\
\text { Weight }\end{array}$ & $\begin{array}{c}\text { Molecular } \\
\text { formula }\end{array}$ \\
\hline 1 & 2.45 & Ethane, 1,1,2-trimethoxy- & 19.02 & 120 & C5H12O3 \\
\hline 2 & 2.50 & d-(+)-Glyceric acid & 15.57 & 106 & $\mathrm{C} 3 \mathrm{H} 6 \mathrm{O} 4$ \\
\hline 3 & 2.62 & 2-Butanol, 1-methoxy- & 30.18 & 104 & $\mathrm{C} 5 \mathrm{H} 12 \mathrm{O} 2$ \\
\hline 4 & 33.85 & Oleic Acid & 29.15 & 282 & $\mathrm{C} 18 \mathrm{H} 34 \mathrm{O} 2$ \\
\hline 5 & 33.94 & 11-Octadecenoic acid, methyl ester & 6.07 & 296 & $\mathrm{C} 19 \mathrm{H} 36 \mathrm{O} 2$ \\
\hline
\end{tabular}

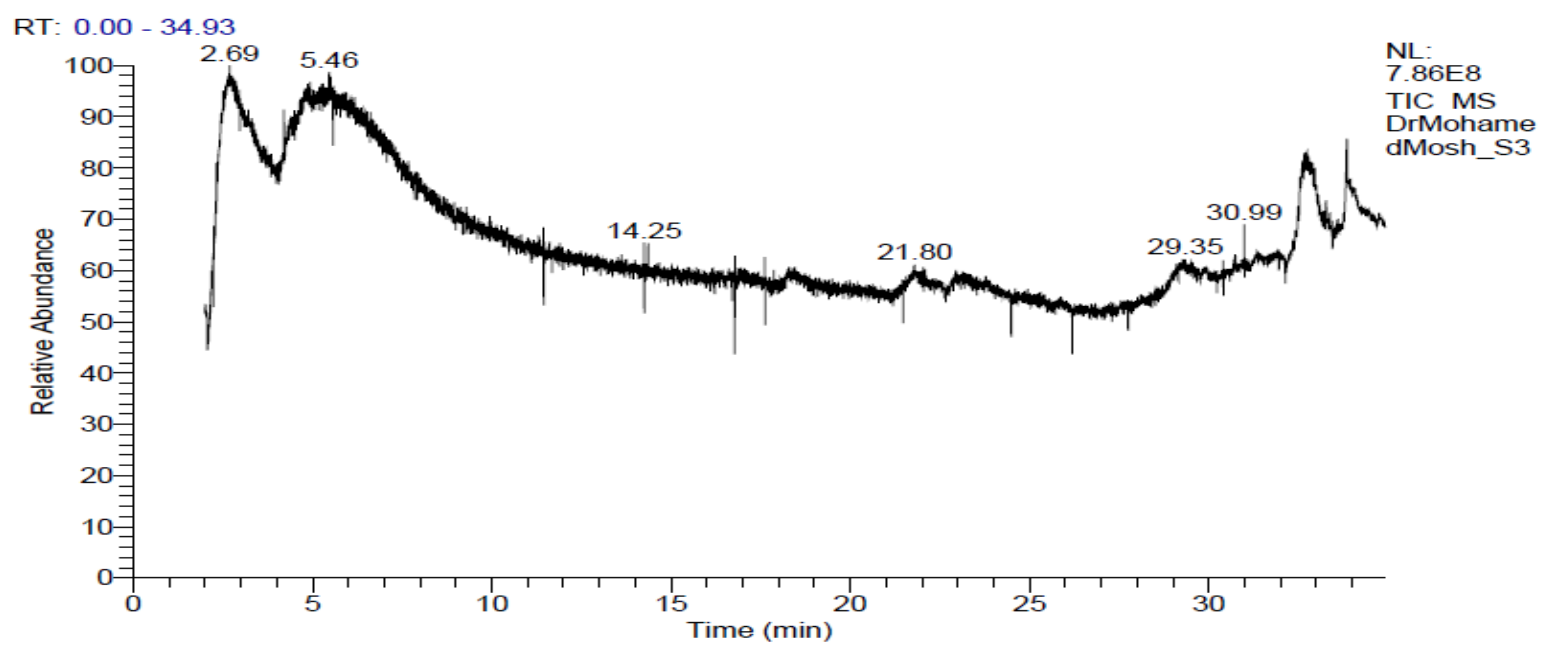

Fig (11): Volatile compounds identified in headspace of fresh mango blending with apricot juice by GC/ MS chromatography analysi.

\section{CONCLUSIONS}

\section{References}

A.O.A.C. (2016). Association of Official Analytical Chemists. Official Methods of Analysis. $\left(20^{\text {th }} \mathrm{Ed}\right.$.) Maryland, USA.

Abu el- maaty, A.S.M (2012). studies on quality properties of some fruit juices. Msc. Thesis, Fac. Agric,. Benha Univ., Egypt

Ajila, C.M.; Naidu, K.A.; Bhat, S.G.; Prasada Rao, U.J.S.( 2007 ). Bioactive compounds and antioxidant potential of mango peel extract. Food Chem., 105, 982-988.

Aroujalian,A., and Raisi,A.(2007).Recovery of volatile aroma components from orange juice by pervaporation. Journal of Membrane Science ,303, 154-161.
Averbeck, M., and Schieberle, P. (2011). Influence of different storage conditions on changes in the key aroma compounds of orange juice reconstituted from concentrate. European Food Research and Technology, 232(1), 129-142.

Azodanlou, R.; Darbellay, C.; Luisier, J.L.; Villettaz, J.C. and Amad`o, R. (2003). Development of a model for quality assessment of tomatoes and apricots, Lebensmittel-Wissenschaft Technol. 36 223-233.

Baietto, M. and Wilson, A. D. (2015). ElectronicNose Applications for Fruit Identification, Ripeness and Quality Grading. Sensors, 15, 899931. www.mdpi.com/journal/sensors.

Cerdán-Calero, M., Izquierdo, L., and Sentandreu, E. (2013). Valencia Late orange juice preserved by pulp reduction and high pressure homogenization: Sensory quality and gas chromatography-mass spectrometry analysis of volatiles. LWT-Food Science and Technology, 51(2), 476-483. 
Chauhan, S., Tyagi, S. and Singh, D., (2001). Pectinolytic liquefaction of apricot, plum, and mango pulps for juice extraction. Int. J. Food Prop. 4 (1), 103_109.

Evrendilek, G. A.(2016): Change regime of aroma active compounds in response to pulsed electric field treatment time, sour cherry juice apricot and peach nectars, and physical and sensory properties. Innovative Food Science and Emerging Technologies $33:$ 195-205.

FAO. (2013). Food and Agriculture Organization of the United Nations. URL <http://faostat3.fao.org/browse/Q/QD/E > . Accessed 15.05.15.

Fukuda, T., Okazaki, K., and Shinano, T. (2013). Aroma characteristic and volatile profiling of carrot varieties and quantitative role of terpenoid compounds for carrot sensory attributes. Journal of food science, $78(11)$.

Göğüş, F., Özel, M. Z., and Lewis, A. C. (2007). The effect of various drying techniques on apricot volatiles analysed using direct thermal desorptionGC-TOF/MS. Talanta, 73(2), 321-325.

Guillot, S. ;Peytavi,L.; Bureau,S ;Boulanger,. R. ;Lepoutre,J.P. ;Crouzet,J. and SchorrGalindo, S. (2006) Aroma characterization of various apricot varieties using headspace-solid phase microextraction combined with gas chromatography-mass spectrometry and gas chromatographyolfactometry, Food Chem. 96 147-155.

John, K. S., Rao, L. J. M., Bhat, S. G., and Rao, U. P. (1999). Characterization of aroma components of sap from different Indian mango varieties. Phytochemistry, 52(5), 891-894.

Khakimov, B., Mongi, R. J., Sørensen, K. M., Ndabikunze, B. K., Chove, B. E., and Engelsen, S. B. (2016). A comprehensive and comparative GC-MS metabolomics study of non-volatiles in Tanzanian grown mango, pineapple, jackfruit, baobab and tamarind fruits. Food chemistry, 213, 691-699.

Kjeldsen, F., Christensen, L. P., and Edelenbos, M. (2001). Quantitative analysis of aroma compounds in carrot (Daucus carota L.) cultivars by capillary gas chromatography using large-volume injection technique. Journal of agricultural and food chemistry, 49(9), 4342-4348.

Kreutzmann, S., Thybo, A. K., Edelenbos, M., and Christensen, L. P. (2008). The role of volatile compounds on aroma and flavour perception in coloured raw carrot genotypes. International journal of food science \& technology, 43(9), 16191627.

Markstrat (2013). Consumo de suco de laranja 40 principais mercados. Retrieved from: http://www.citrusbr.com/download/ (Accessed on 02 August 2013).

Mastello, R. B., Capobiango, M., Chin, S. T., Monteiro, M., \& Marriott, P. J. (2015).
Identification of odour-active compounds of pasteurised orange juice using multidimensional gas chromatography techniques. Food Research International, 75, 281-288.

Mihalev, K., Schieber, A., Mollov, P., Carle, R., (2004). Effect of mash maceration on the polyphenolic content and visual quality attributes of cloudy apple juice. J. Agric. Food Chem. 52, 7306_7310.

Musharraf, S. G., Uddin, J., Siddiqui, A. J., and Akram, M. I. (2016). Quantification of aroma constituents of mango sap from different Pakistan mango cultivars using gas chromatography triple quadrupole mass spectrometry. Food chemistry, 196, 1355-1360.

Nigam, P.S., Pandey, A., (2009). Biotechnology for Agro-Industrial Residues Utilization. Springer, Netherlands

Qiao, Y., Xie, B. J., Zhang, Y., Zhang, Y., Fan, G., Yao, X. L., and Pan, S. Y. (2008). Characterization of aroma active compounds in fruit juice and peel oil of Jinchen sweet orange fruit (Citrus sinensis (L.) Osbeck) by GC-MS and GC-O. Molecules, 13(6), 1333-1344.

Ramteke, R.S.; Eipeson, W.E. and Patwardhan, M.V. (1990) Behavior of aroma volatiles during the evaporative concentration of some tropical fruit juices and pulps, J. Sci. Food Agric. 50 (3) 399.

Riu-Aumatell, M., Castellari, M., LópezTamames, E., Galassi, S., and Buxaderas, S. (2004). Characterisation of volatile compounds of fruit juices and nectars by HS/SPME and GC/MS. Food Chemistry, 87(4), 627-637.

Rizzolo, A.; Polesello, A. and Polesello, S. (2002): Use of headspace capillary G. C. to study the development of volatile compounds in fresh fruits. J. of High. Resol-chromatogrm, 15:472-477.

Schwab, W.; Davidovich-Rikanati, R.; Lewinsohn, E. (2008).Biosynthesis of plant-derived flavor compounds. Plant J., 54, 712-732.

Selli, S., and Kelebek, H. (2011). Aromatic profile and odour-activity value of blood orange juices obtained from Moro and Sanguinello (Citrus sinensis L. Osbeck). Industrial Crops and Products, 33(3), 727-733.

Selli, S., Cabaroglu, T., and Canbas, A. (2004). Volatile flavour components of orange juice obtained from the cv. Kozan of Turkey. Journal of Food Composition and Analysis, 17(6), 789-796.

Sharoba, A.M.; Bahlol, H.E.M. and El-Desouky, A. I (2007). Establishing a schedule to determine the optimal thermal process time for some canned fruit products . Annals Of Agric. Sc., Moshtohor, Vol. 45(1): 125-145.

Sharma, B.R., Naresh, L., Dhuldhoya, N.C., Merchant, S.U., Merchant, U.C., (2006). An overview on pectins. Times Food Process. J. June_July, 44_51. 
Sharma, H.P., Patel, H., Sharma, S.,(2014). Enzymatic extraction and clarification of juice from various fruits_a review. Trends Post Harvest Technol. 2 (1), 01_14.

Sharma, H.P., Patel, H., Sugandha, (2017). Enzymatic added extraction and clarification of fruit juices _ a review. Crit. Rev. Food Sci. Nutr. 57 (6), 1215_1227. Available from: http://dx.doi.org/10.1080/ 10408398.2014.977434.

Singh, S. P., and Saini, M. K. (2014). Postharvest vapour heat treatment as a phytosanitary measure influences the aroma volatiles profile of mango fruit. Food chemistry, 164, 387-395.

Solis-Solis, H. M., Calderon-Santoyo, M., Gutierrez-Martinez, P., Schorr-Galindo, S., and Ragazzo-Sanchez, J. A. (2007). Discrimination of eight varieties of apricot (Prunus armeniaca) by electronic nose, LLE and SPME using GC-MS and multivariate analysis. Sensors and Actuators B: Chemical, 125(2), 415-421.

Solís-Solís, H. M., Calderón-Santoyo, M., SchorrGalindo, S., Luna-Solano, G., and RagazzoSánchez, J. A. (2007). Characterization of aroma potential of apricot varieties using different extraction techniques. Food chemistry, 105(2), 829-837.

Tharanathan, R. N., Yashoda, H. M., and Prabha, T. N. (2006). Mango (Mangifera indica L.), "The King of Fruits"-An overview. Food Reviews International, 22(2), 95-123.

Theodosiou M, Laudet V, Schubert M. 2010. From carrot to clinic: an overview of the retinoic acid signaling pathway. Cell Mol Life Sci 67:1423-45.

Tucker, G.A. Introduction. In Biochemistry of Fruit Ripening; Seymour, G.B., Taylo, R.J.E., Tucker, G.A., Eds.; Chapman \& Hall: London, UK, 1993; pp. 1-51.

Vervoort, L., Grauwet, T., Kebede, B. T., Van der Plancken, I., Timmermans, R., Hendrickx, M., and Van Loey, A. (2012). Headspace fingerprinting as an untargeted approach to compare novel and traditional processing technologies: a case-study on orange juice pasteurisation. Food chemistry, 134(4), 23032312.

Zhu W.; Zhu B.; Li Y.; Zhang Y., ; Zhang B., and Fan J. (2016): Acidic electrolyzed water efficiently improves the flavour of persimmon (Diospyros kaki L. cv. Mopan) wine. Food Chemistry 197 : 141-149. 


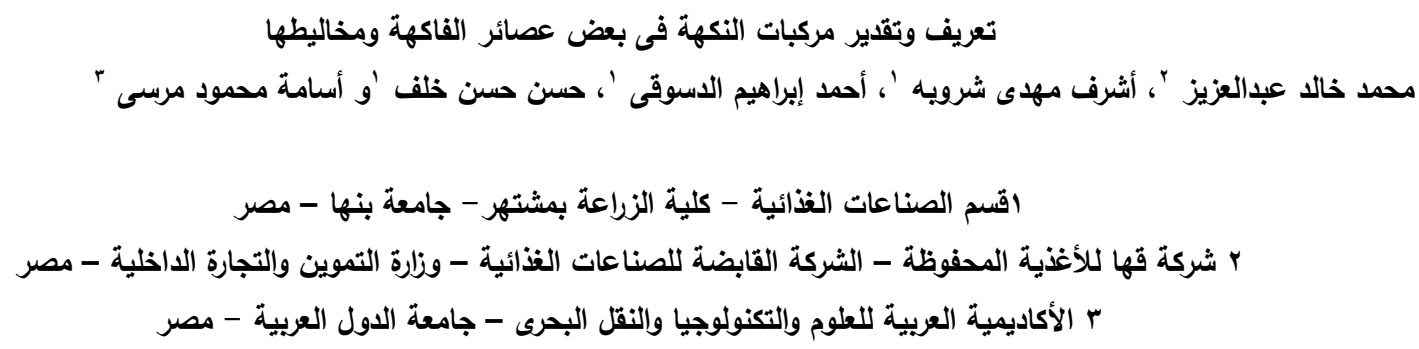

في هذه الدراسـة نم استخدام طريقة النحليل الكروموتوجرفي لمعرفة الككونات المسئولة عن النكهة في بعض أنواع العصـائر والمشروبات

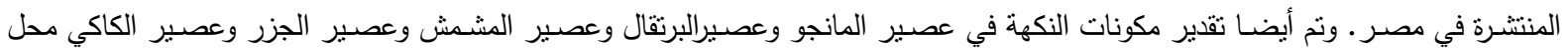

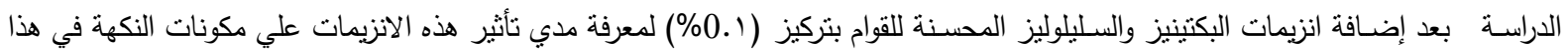

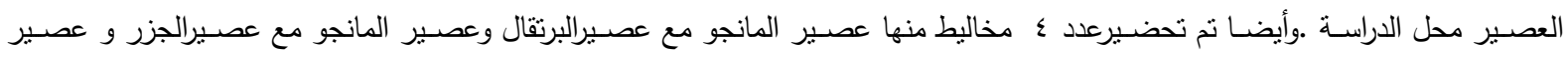

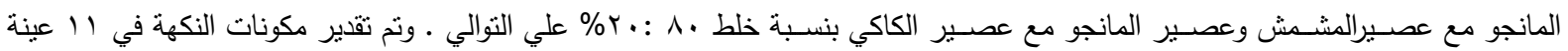

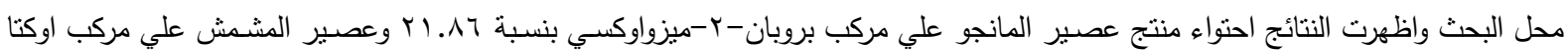

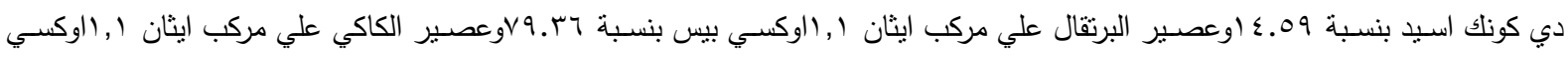

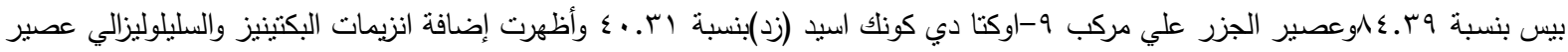

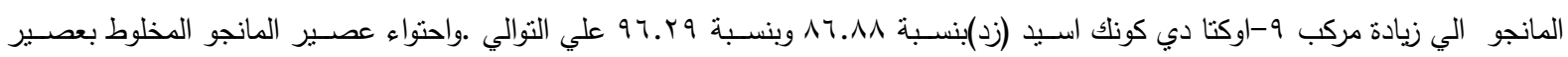

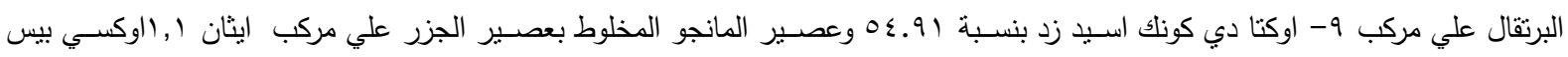

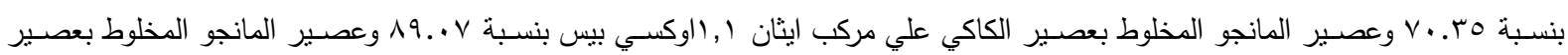

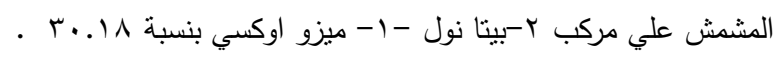

